\title{
Aerosol-radiation interaction in atmospheric models: Idealized sensitivity study of simulated short-wave direct radiative effects to particle microphysical properties
}

\author{
V. Obiso*, O. Jorba \\ Earth Sciences Department, Barcelona Supercomputing Center (BSC-ES), c/ Jordi Girona 29, 08034 Barcelona, Spain
}

\section{A R T I C L E I N F O}

\section{Keywords:}

Aerosol microphysical properties

Aerosol optical properties

Radiative transfer simulations

Aerosol-radiation interaction

Aerosol direct radiative effects

\begin{abstract}
A B S T R A C T
We assessed the impact of the microphysical parameterization of natural and anthropogenic aerosols on simulated short-wave radiative effects due to Aerosol-Radiation Interaction (ARI). Layer radiative properties (optical depth, single scattering albedo and asymmetry factor) of dry mineral dust, organic carbon and a black carbon-sulfate mixture have been calculated with a Tmatrix code in the short-wave spectral region, after perturbing relevant particle microphysical properties (size distribution, refractive index, mixing state). For each aerosol species, an idealized atmospheric layer and three events of increasing intensity have been set. Then, short-wave direct radiative effects (clear-sky) have been simulated at the top-of-atmosphere (TOA) and at surface (SFC) using the radiative transfer model RRTMG_SW (widely used in atmospheric models), separately for each aerosol species. We observed considerably variable impacts of the particle microphysical perturbations on the layer radiative properties for mineral dust and organic carbon, mainly due to the different sizes of the two species. For the black carbon-sulfate mixture, the single scattering albedo has been found to be much lower in the internal mixing case. Regarding the direct radiative effects, we observed perturbation-induced variability ranges (evaluated against the base net fluxes in absence of aerosols) always within the perturbation range set for the particle microphysical properties $( \pm 20 \% \rightarrow 40 \%)$. This work, therefore, quantitatively demonstrates that small uncertainties on the aerosol microphysical parameterization propagate on the simulated direct radiative effects mainly with a loss of strength. Considerable perturbation-induced absolute variations of the direct radiative effects have been found (above all for large aerosol amounts), which could significantly affect the model assessments of the ARI radiative effects and therefore meteorological forecasts and climate predictions.
\end{abstract}

\section{Introduction}

Atmospheric aerosols can redistribute the radiative energy in the atmosphere through scattering and absorption of electromagnetic radiation (Boucher et al., 2013). The Aerosol-Radiation Interaction (ARI), as one of the several climate forcing agents, still contributes to dominate the significant uncertainty affecting the anthropogenic contribution to the climate change (Myhre et al., 2013b), even if its parameterization in atmospheric models has strongly improved in the last two decades (Myhre et al., 2013a). Also the role of the natural aerosols in affecting the Earth's radiative balance through ARI is poorly constrained (Rap et al., 2013). The ARI features are mainly defined by the characterization of the aerosol optical properties. Yu et al. (2006) reviewed several measurement-

\footnotetext{
* Corresponding author.

E-mail addresses: vincenzo.obiso@bsc.es (V. Obiso), oriol.jorba@bsc.es (O. Jorba).
} 
based assessments of ARI radiative effects (from ground-based networks, satellite remote sensing and intensive field experiments), supported by chemical transport model simulations. They pointed out that still large uncertainties in the estimates of the ARI radiative effects exist and deduced that errors in the estimation of the aerosol optical properties can have a tremendous impact on the assessments of the ARI radiative effects.

The aerosol optical properties for model simulations are calculated with optical codes starting from particle microphysical properties, such as size distribution, refractive index, mixing state, mass density, shape and hygroscopicity. Hence, different particle microphysical assumptions can affect the calculation of the optical properties and therefore the model assessments of the ARI radiative effects. In the last two decades, many sensitivity studies of simulated radiative fluxes to aerosol microphysical and optical properties have been done. However, in most works the analysis has been mainly carried out on single aerosol species, either mineral dust (Liao \& Seinfeld, 1998; Tegen \& Lacis, 1996), carbonaceous aerosols (Bauer, Menon, Koch, Bond, \& Tsigaridis, 2010), sulfate (Nemesure, Wagener, \& Schwartz, 1995) or black carbon-sulfate mixture (Lesins, Chylek, \& Lohmann, 2002). Moreover, different microphysical parameters with very different experiment setups (perturbations considered, simulation configurations, analysis methods, etc.) have been investigated. Hence, even if all these works agree that the microphysical assumptions can have relevant impacts on the ARI radiative effect simulations, it appears to be difficult to find results relative to different properties or species that are directly comparable.

In this paper we present a systematic and detailed study on the propagation of particle microphysical perturbations, applied to several microphysical parameters of natural and anthropogenic aerosol species, on the optical and radiative simulations usually required by the ARI implementation in weather and climate models. Our analysis, therefore, has been carried out at two levels: (i) impact of the particle microphysical perturbations on layer radiative properties and (ii) consequent impact on short-wave direct radiative effects, at the top-of-atmosphere (TOA) and at surface (SFC). Since our work mainly aimed to provide results suitable for atmospheric model simulations, we used aerosol databases and numerical tools widely used in the weather and climate modeling community. Four important global aerosol species that are usually considered in aerosol models have been separately investigated: mineral dust and organic carbon, in order to study particles with different sizes (producing results suitable in a certain degree for other species with similar sizes), and black carbon and sulfate, in order to include in the analysis the particle mixing state. All the particles have been considered spherical, homogeneous and in dry conditions. Local perturbations have been applied to the size distribution and refractive index parameters for mineral dust and organic carbon. The perturbation range ( $\pm 20 \%)$ has been set to represent, in a first approximation, uncertainties affecting the aerosol microphysical parameters. Our main objective, however, was not to accurately represent realistic experimental uncertainties but to consider a homogeneous (and reasonable) perturbation range for all the species and microphysical properties. On the other hand, for the black carbon-sulfate mixture two different mixing states (internal and external) have been considered. Starting from these perturbed microphysical assumptions, layer optical depth, single scattering albedo and asymmetry factor have been calculated for all the species and their perturbation-induced relative spreads evaluated in all the short-wave region.

Following Liao and Seinfeld (1998), we then performed one-column radiative transfer simulations with and without activating idealized aerosol layers (short-wave and clear-sky conditions) in order to separately calculate direct radiative effects (at the TOA and at SFC) under perturbations of different properties and aerosol species. Again, we did not aim to simulate realistic direct radiative effects (for which mass transport simulations with integrated meteorology-chemistry models are needed) but to focus the analysis on the radiative sensitivity to the particle microphysical perturbations. Three increasing values of mass concentration for each species have been set in the homogeneous layers in order to simulate aerosol events of increasing intensity. In order to provide results suitable for general atmospheric scenarios (for example different meteorological and geographical assumptions), the perturbationinduced variability ranges of the direct radiative effects have been evaluated against the base net fluxes in absence of aerosols.

This work represents the second part of the paper Obiso, Pandolfi, Ealo, and Jorba (2017), in which we analyzed the sensitivity of mass scattering cross sections of three important aerosol species (mineral dust, organic carbon and sulfate) to perturbed microphysical properties (size distribution, refractive index, mass density and shape) and derived constraints on these microphysical properties from a comparison between simulated and observed aerosol scattering properties. This paper is organized as follows: in Section 2, a detailed description of the numerical tools used is given; in Section 3, the setup details for all the simulations are explained; in Sections 4 and 5, the results of the experiment are presented (separately for each aerosol species) and discussed, respectively; in Section 6, the conclusions about the work are presented.

\section{Methodology}

The study presented in this paper required the use of an optical code and a radiative transfer model suitable for applications in weather and climate models. An optical code is able to calculate scattering and absorption of radiation either by a mono-disperse (only one size) or poly-disperse particles (different distributed sizes), starting from their microphysical properties. A radiative transfer model performs computations of radiative fluxes given an atmospheric structure, gas and aerosol profiles and optical properties. In this section we briefly describe the numerical tools used in this work.

\subsection{T-matrix code}

The T-matrix method is a powerful exact technique for computing light scattering by non-spherical particles (Mishchenko \& Travis, 1998). The incident and the scattered electromagnetic fields are expanded in series of vector spherical wave functions, with the relation between the expansion coefficients established through the T-matrix (Mishchenko, Videen, Babenko, 
Khlebtsov, \& Wriedt, 2004). The T-Matrix is determined only by the particle microphysical properties (size parameter, aspect ratio, refractive index) and for a given incident field it allows calculating the scattered field, the scattering matrix and the optical cross sections (Mishchenko \& Travis, 1998).

For our experiment the T-matrix code by Mishchenko, Travis, and Lacis (2002) has been used. It allows calculating integrated optical properties of poly-disperse, randomly oriented, rotationally symmetric, homogeneous particles. In particular, spectral extinction efficiency $\left(Q_{\lambda}^{\text {ext }}\right)$, single scattering albedo $\left(\omega_{\lambda}\right)$ and asymmetry factor $\left(g_{\lambda}\right)$ (optical properties of interest for the radiative flux simulations) are calculated as follows (Mishchenko, Travis, \& Lacis, 2002):

$$
\begin{aligned}
Q_{\lambda}^{e x t} & =\frac{\int Q_{\lambda}^{e x t}(r) \pi r^{2} f(r) d r}{\int \pi r^{2} f(r) d r}, \\
\omega_{\lambda} & =\frac{\int \omega_{\lambda}(r) Q_{\lambda}^{\text {ext }}(r) \pi r^{2} f(r) d r}{\int Q_{\lambda}^{e x t}(r) \pi r^{2} f(r) d r}, \\
g_{\lambda} & =\frac{\int g_{\lambda}(r) Q_{\lambda}^{s c a}(r) \pi r^{2} f(r) d r}{\int Q_{\lambda}^{s c a}(r) \pi r^{2} f(r) d r},
\end{aligned}
$$

where $Q_{\lambda}^{\text {ext }}(r), \omega_{\lambda}(r)$ and $g_{\lambda}(r)$ are the optical properties of a single particle of radius $r$ and $f(r)$ is the normalized particle number size distribution (log-normal in our case). The single-particle single scattering albedo is defined as the ratio of scattering $\left(Q_{\lambda}^{s c a}\right)$ to extinction efficiency:

$$
\omega_{\lambda}(r)=\frac{Q_{\lambda}^{s c a}(r)}{Q_{\lambda}^{e x t}(r)},
$$

and represents a measure of the fractional amount of scattered energy with respect to the total extinct energy (always $\omega_{\lambda} \leq 1$ ). The single-particle asymmetry factor is defined as the first moment of the scattering phase function $\left(P_{\lambda}\right)$ :

$$
g_{\lambda}(r)=\frac{1}{4 \pi} \int_{4 \pi} P_{\lambda}(\Theta) \cos \Theta d \Omega
$$

where $\Theta$ is the scattering angle. It gives a measure of the scattered energy in the forward direction with respect to the total scattered energy $(0<g \leq 1$ for scattering mainly in the forward direction and $g=0$ for isotropic scattering) (Mishchenko, Travis, \& Lacis, 2002).

For "standard" particle microphysical parameters (not too extreme sizes or aspect ratios), the T-matrix code is able to produce very accurate results (five and more exact decimal places). Moreover, it has an automatic built-in convergence test which provides a very good estimate of the absolute accuracy of the computations (Mishchenko \& Travis, 1998).

\subsection{RRTMG_SW model}

The RRTMG_SW (Iacono et al., 2008) is a 1-D rapid and accurate radiative transfer model suitable for global circulation models and mesoscale/regional models (Iacono, 2011). It is based on the RRTM_SW (Clough et al., 2005), an adaptation to short-wave of the RRTM (Rapid Radiative Transfer Model) by Mlawer, Taubman, Brown, Iacono, and Clough (1997). The RRTM utilizes the $k$-correlated method (Fu \& Liou, 1992), an approximation technique to perform accelerated calculation of radiative fluxes with an extreme reduction of the number of operations. Given a homogeneous layer and a spectral band (conveniently defined to take into account the most important spectrally active gaseous species), the radiative transfer operations are carried out using a small set of absorption coefficients (taken from the LBLRTM model (Clough \& Iacono, 1995; Clough, Iacono, \& Moncet, 1992)) representative of all the band. This is possible thanks to a mapping of the absorption coefficients from the wavenumber space onto a cumulative probability space, defined by the variable $g$ (fraction of absorption coefficients smaller than a certain value). In this new space the absorption coefficients have a regular increasing trend and so a mean value can be specified for each segment ( $g$-point) of this smoothly varying function (Mlawer, Taubman, Brown, Iacono, \& Clough, 1997). Respect to the RRTM_SW, the RRTMG_SW includes some new features in order to work as a callable routine of weather and climate models, such as a reduced number of $g$-points, a Montecarlo method (McICA (Barker, Pincus, \& Morcrette, 2003)) to represent sub-grid cloud variability, new aerosol input flags and a new faster twostream algorithm for multiple scattering radiative transfer (Oreopoulos \& Barker, 1999). Many two-stream methods are available in literature, derived with slight different approximations (Meador \& Weaver, 1980), but all are based on reducing the general radiative transfer equations for the spectral radiance to the following couple of differential equations for spectral diffuse upward $\left(F_{d i f, \lambda}^{\uparrow}\right)$ and downward $\left(F_{d i f, \lambda}^{\downarrow}\right)$ fluxes (replacing the solar source term with a thermal emission term would give the equations for long-wave) (Jacobson, 2005):

$$
\begin{gathered}
\frac{d F_{d i f, \lambda}^{\uparrow}(\tau)}{d \tau}=\gamma_{1} F_{d i f, \lambda}^{\uparrow}(\tau)-\gamma_{2} F_{d i f, \lambda}^{\downarrow}(\tau)-\gamma_{3} \omega_{\lambda} F_{d i r, \lambda}(\tau), \\
-\frac{F_{d i f, \lambda}^{\downarrow}(\tau)}{d \tau}=\gamma_{1} F_{d i f, \lambda}^{\downarrow}(\tau)-\gamma_{2} F_{d i f, \lambda}^{\uparrow}(\tau)-\left(1-\gamma_{3}\right) \omega_{\lambda} F_{d i r, \lambda}(\tau),
\end{gathered}
$$

in which $F_{d i r, \lambda}(\tau)=F_{s, \lambda} \exp \left(-\tau / \mu_{s}\right)$ is the direct flux, that is what remains of the solar flux at the TOA $\left(F_{s, \lambda}\right)$ after the atmospheric extinction, with $\mu_{s}$ being the cosine of the solar zenith angle (SZA), and the $\gamma_{i}$ are in general functions of single scattering albedo, 
Table 1

Wavelength intervals $(\Delta \lambda)$ of the RRTMG_SW spectral bands.

\begin{tabular}{ll}
\hline Band Number (b) & $\Delta \lambda(\mu m)$ \\
\hline 1 & $0,200-0,263$ \\
2 & $0,263-0,345$ \\
3 & $0,345-0,442$ \\
4 & $0,442-0,625$ \\
5 & $0,625-0,778$ \\
6 & $0,778-1,242$ \\
7 & $1,242-1,299$ \\
8 & $1,299-1,626$ \\
9 & $1,626-1,942$ \\
10 & $1,942-2,151$ \\
11 & $2,151-2,500$ \\
12 & $2,500-3,077$ \\
13 & $3,077-3,846$ \\
14 & $3,846-12,195$ \\
\hline
\end{tabular}

asymmetry factor and $S Z A$, determined by the particular two-stream approximation used (Meador \& Weaver, 1980). In particular, $\gamma_{1}$ takes into account layer extinction and forward scattering of the diffuse fluxes (so includes a loss and a gain term), $\gamma_{2}$ the backward scattering of the diffuse fluxes (gain term) and $\gamma_{3}$ the backward scattering of the direct flux (gain term), being (1 $-\gamma_{3}$ ) the forward scattering term.

For our experiment we used the RRTMG_SW in the stand-alone version. The model divides the short-wave region $(0,200<\lambda<12,195 \mu \mathrm{m})$ in fourteen spectral bands, reported in Table 1, and allows considering the presence of aerosol layers in atmosphere by requiring as inputs layer optical depth, single scattering albedo and asymmetry factor for each of these spectral bands. The RRTMG_SW has been designed to be run in atmospheric model simulations and so to perform rapid calculations in order to save computation time. Obviously, these characteristics (and all the underlying approximations) cause the calculation precision to be slightly lower than that of more complex line-by-line or 3-D radiative transfer models available in literature. However, the RRTMG_SW has an accuracy of 0, 3\% (clear-sky) compared to the RRTM_SW, the RRTM_SW of $\sim 0,3 \%$ (clear-sky) compared to the LBLRTM (direct plus diffuse fluxes) and the LBLRTM of $\sim 0,5 \%$ compared to observations (Iacono et al., 2008). Hence, the RRTMG_SW retains a good total accuracy of $\sim 1 \%$ with respect to observations. Moreover, the RRTMG_SW is used in many important models (a list of global and regional model applications is reported in Iacono (2011)) and therefore it has been considered an appropriate tool for our sensitivity study.

\section{Experiment setup}

This section presents the experiment setup in three parts: (i) definition of aerosol reference and perturbed microphysical properties, (ii) calculation of aerosol optical and layer radiative properties, (iii) simulation of direct radiative effects at the TOA and at SFC.

\subsection{Reference aerosol species and microphysical perturbations}

Four important global aerosol species have been studied in this work: mineral dust, organic carbon, black carbon and sulfate. The following particle microphysical properties have been considered to characterize the reference aerosol species: size distribution (geometric radius $r_{g}[\mu \mathrm{m}]$ and standard deviation $\sigma_{g}$ [unitless]), refractive index (real $n_{r}$ and imaginary $n_{i}$ parts [unitless]) and mass density $\left(\rho\left[\mathrm{kg} / \mathrm{m}^{3}\right]\right)$. All the aerosol species have always been considered spherical, homogeneous and in dry conditions (no relative humidity effects on the particle optical properties have been analyzed). We defined the reference microphysical properties following the aerosol parameterization used in the NMMB-MONARCH model (formerly known as NMMB/BSC-CTM) (Badia et al., 2017; Di Tomaso, Schutgens, Jorba, \& Pérez García-Pando, 2017; Jorba et al., 2012; Pérez et al., 2011; Spada, Jorba, Pérez García-Pando, Janjic, \& Baldasano, 2013), which in turn is based on the OPAC database (Hess, Köpke, \& Schult, 1998) but with some modifications. Mineral dust has been associated with the mineral (accumulation mode) OPAC component (MIAM), except for geometric radius, taken from Shettle (1984), and mass density, taken from Nickovic, Kallos, Papadopoulos, and Kakaliagou (2001) (the NMMBMONARCH model considers different values of dust mass density for sub-micron and super-micron bins; so for our bulk mineral dust we derived a weighted mean of the original values). The dust imaginary refractive index values used (taken from OPAC) are compatible in the visible range with the mean of the experimental estimates reported in Sokolik, Andronova, and Johnson (1993). Organic carbon has been associated with the water-soluble OPAC component (WASO), except for standard deviation, taken from Chin et al. (2002), and imaginary refractive index. Organic carbon is a complex species, since the definition includes organic particles with very different absorbing capabilities (biogenic and anthropogenic organic matter, brown carbon from biomass burnings, etc.). Hence, we modified the OPAC imaginary refractive index values in the visible range in order to make them more compatible with the mean of the experimental estimates reported in Liu et al. (2015). Finally, black carbon has been associated with the soot OPAC component (SOOT) and sulfate with the sulfate-droplets OPAC component (SUSO). 
In order to generate variability in the aerosol microphysical parameterization for the sensitivity study, we perturbed all the reference parameters of the relevant microphysical properties (always in this work marked with ${ }^{(*)}$ ). For mineral dust and organic carbon we multiplied the reference parameters of size distribution $\left(r_{g}^{*}\right.$ and $\sigma_{g}^{*}$ separately) and refractive index $\left(n_{r}^{*}\right.$ and $n_{i}^{*}$ separately) for perturbation factors: $m$ (i.e. minus), $r$ (i.e. reference) and $p$ (i.e. plus). The values $(m=0,8 ; r=1,0 ; p=1,2)$ have been selected for all the parameters. It is important to note that the two microphysical properties have been perturbed separately, so when the size distribution was perturbed the reference refractive index values were used and vice versa. Also, for each microphysical property the two parameters have been perturbed independently but allowing the composite perturbations (in order to expand the perturbation space, as suggested by Zhao et al. (2013)). So nine perturbation cases for each property have been derived. Mass density and shape have not been directly perturbed in this work because in Obiso, Pandolfi, Ealo, and Jorba (2017) they have been found in most cases to be less important than size distribution and refractive index in affecting the aerosol scattering properties. On the other hand, black carbon and sulfate have been considered as a mixture in two different mixing states: external (em) and internal ( $\mathrm{im}$ ). For the em case, the single species reference microphysical properties have been used to separately calculate the spectral optical properties of black carbon and sulfate. These properties have been then coupled a posteriori to derive the layer radiative properties of the externally mixed species, as explained in Section 3.2. For the im case, we supposed at first that all the sulfate mass condenses on the black carbon particles with a constant volume ratio $(R)$ (in the following equations $b c$ and $s u$ indicate black carbon and sulfate, respectively):

$$
R=\frac{V_{b c}}{V_{b c+s u}}
$$

Then, we imposed the conservation of the sulfate mass concentration:

$$
\int\left(V_{b c+s u}-V_{b c}\right) \rho_{s u} N_{b c} f_{b c}(r) d r=M_{s u},
$$

where, in addition to the known symbols, $N$ is the number concentration and $M$ the mass concentration of the particles. Finally, from Eqs. (5) and (6), we derived the following formula for $R$ :

$$
R=\frac{\rho_{s u} M_{b c}}{\rho_{s u} M_{b c}+\rho_{b c} M_{s u}} .
$$

The value $R=0,357$ has been calculated after setting the mass concentrations for black carbon and sulfate (see Section 3.2). Once derived the volume ratio, the microphysical properties of the internally mixed species have been calculated as follows:

$$
\begin{aligned}
r_{g} & =r_{g, b c}^{*} R^{-1 / 3}, \\
\sigma_{g} & =\sigma_{g, b c}^{*}, \\
n_{r} & =n_{r, b c}^{*} R+n_{r, s u}^{*}(1-R), \\
n_{i} & =n_{i, b c}^{*} R+n_{i, s u}^{*}(1-R), \\
\rho & =\rho_{b c}^{*} R+\rho_{s u}^{*}(1-R) .
\end{aligned}
$$

Hence, the internally mixed particles have been modeled as homogeneous particles with mean refractive index and mass density (so well-mixed particles). The size distribution has been derived by shifting that of black carbon according to the fixed volume ratio, in order to take into account the size increase due to the internal mixing with sulfate. Moreover, with this procedure the total mass concentration of the two species results conserved while the number concentration of the internally mixed species equals that of black carbon.

In Table 2 reference and perturbed size distribution and refractive index values (also due to the different mixing states) for all the species considered are reported. The perturbation cases for mineral dust and organic carbon are named starting from the perturbation factors $m, r$ and $p$ defined above: the first factor refers to $r_{g}$ and $n_{r}$ while the second one to $\sigma_{g}$ and $n_{i}$, for size distribution and refractive index, respectively. The $r r$ cases (i.e. the reference cases) for the two properties are the same case, which we named ref in order to avoid confusion. Regarding the black carbon-sulfate mixture, the external mixing case (em) has been considered as the reference case in the sensitivity analysis while the internal mixing case $(\mathrm{im})$ as the perturbation case. A log-normal size distribution, over a size range $P M_{10}$ (starting from $0,001 \mu \mathrm{m}$ ), has been considered for all the species. For each couple of size distribution parameters, the corresponding effective radius $\left(r_{e f f}\right)$ is also reported (for poly-disperse particles $r_{\text {eff }}$ can be considered as the representative size of the ensemble (Hansen \& Travis, 1974)), which is defined as the projected-area-weighted mean radius (Mishchenko, Travis, \& Lacis, 2002) and can be easily calculated as follows (Lesins, Chylek, \& Lohmann, 2002):

$$
r_{e f f}=r_{g} \exp \left(\frac{5}{2} \ln ^{2} \sigma_{g}\right)
$$

As already mentioned, the mass density has not been directly perturbed in this work but it affects the aerodynamic diameter involved in the definition of the $P M_{10}$ size range (DeCarlo, Slowik, Worsnop, Davidovits, \& Jimenez, 2004).

\subsection{Aerosol optical and layer radiative properties}

Spectral extinction efficiency, single scattering albedo and asymmetry factor of mineral dust, organic carbon (for all the perturbation cases), black carbon, sulfate and the internally mixed species have been calculated with the T-matrix code (Eqs. (1)), 
Table 2

Reference and perturbed microphysical properties for mineral dust, organic carbon and the black carbon-sulfate mixture: geometric radius $\left(r_{g}\right)$ and standard deviation $\left(\sigma_{g}\right)$, real $\left(n_{r}\right)$ and imaginary $\left(n_{i}\right)$ refractive indices. For simplicity, only the refractive index values at $\lambda=0$, $550 \mu \mathrm{m}$ have been reported. The reference mass densities $(\rho)$ used for the species considered are (in $\mathrm{kg} / \mathrm{m}^{3}$ ): $2,506 \cdot 10^{3}$ (mineral dust), $1,800 \cdot 10^{3}$ (organic carbon), $1,000 \cdot 10^{3}$ (black carbon) and $1,700 \cdot 10^{3}$ (sulfate). For the internally mixed species a value of $1,450 \cdot 10^{3}$ has been derived from the last of Eqs. ( 8 ). The perturbation case acronyms have been defined in Section 3.1 .

\begin{tabular}{|c|c|c|c|c|c|c|}
\hline \multirow[b]{2}{*}{ Perturbation Cases } & \multicolumn{3}{|c|}{ Size Distribution } & \multicolumn{3}{|c|}{ Refractive Index } \\
\hline & Parameters & Mineral Dust & Organic Carbon & Parameters & Mineral Dust & Organic Carbon \\
\hline \multirow[t]{3}{*}{ ref } & $r_{g}^{*}(\mu m)$ & $2,986 \cdot 10^{-1}$ & $2,120 \cdot 10^{-2}$ & & & \\
\hline & $\sigma_{g}^{*}$ & 2,000 & 2,200 & $n_{r}^{*}$ & 1,530 & 1,530 \\
\hline & $r_{e f f}^{*}(\mu m)$ & $9,205 \cdot 10^{-1}$ & $1,003 \cdot 10^{-1}$ & $n_{i}^{*}$ & $5,500 \cdot 10^{-3}$ & $6,000 \cdot 10^{-3}$ \\
\hline \multirow[t]{3}{*}{$m m$} & $r_{g}(\mu m)$ & $2,389 \cdot 10^{-1}$ & $1,696 \cdot 10^{-2}$ & & & \\
\hline & $\sigma_{g}$ & 1,600 & 1,760 & $n_{r}$ & 1,224 & 1,224 \\
\hline & $r_{e f f}(\mu m)$ & $4,150 \cdot 10^{-1}$ & $3,771 \cdot 10^{-2}$ & $n_{i}$ & $4,400 \cdot 10^{-3}$ & $4,800 \cdot 10^{-3}$ \\
\hline \multirow[t]{3}{*}{$m r$} & $r_{g}(\mu m)$ & $2,389 \cdot 10^{-1}$ & $1,696 \cdot 10^{-2}$ & & & \\
\hline & $\sigma_{g}$ & 2,000 & 2,200 & $n_{r}$ & 1,224 & 1,224 \\
\hline & $r_{e f f}(\mu m)$ & $7,617 \cdot 10^{-1}$ & $8,024 \cdot 10^{-2}$ & $n_{i}$ & $5,500 \cdot 10^{-3}$ & $6,000 \cdot 10^{-3}$ \\
\hline \multirow[t]{3}{*}{$m p$} & $r_{g}(\mu m)$ & $2,389 \cdot 10^{-1}$ & $1,696 \cdot 10^{-2}$ & & & \\
\hline & $\sigma_{g}$ & 2,400 & 2,640 & $n_{r}$ & 1,224 & 1,224 \\
\hline & $r_{e f f}(\mu m)$ & 1,150 & $1,782 \cdot 10^{-1}$ & $n_{i}$ & $6,600 \cdot 10^{-3}$ & $7,200 \cdot 10^{-3}$ \\
\hline \multirow[t]{3}{*}{$r m$} & $r_{g}(\mu m)$ & $2,986 \cdot 10^{-1}$ & $2,120 \cdot 10^{-2}$ & & & \\
\hline & $\sigma_{g}$ & 1,600 & 1,760 & $n_{r}$ & 1,530 & 1,530 \\
\hline & $r_{e f f}(\mu m)$ & $5,187 \cdot 10^{-1}$ & $4,713 \cdot 10^{-2}$ & $n_{i}$ & $4,400 \cdot 10^{-3}$ & $4,800 \cdot 10^{-3}$ \\
\hline \multirow[t]{3}{*}{$r p$} & $r_{g}(\mu m)$ & $2,986 \cdot 10^{-1}$ & $2,120 \cdot 10^{-2}$ & & & \\
\hline & $\sigma_{g}$ & 2,400 & 2,640 & $n_{r}$ & 1,530 & 1,530 \\
\hline & $r_{e f f}(\mu m)$ & 1,293 & $2,219 \cdot 10^{-1}$ & $n_{i}$ & $6,600 \cdot 10^{-3}$ & $7,200 \cdot 10^{-3}$ \\
\hline \multirow[t]{3}{*}{$p m$} & $r_{g}(\mu m)$ & $3,583 \cdot 10^{-1}$ & $2,544 \cdot 10^{-2}$ & & & \\
\hline & $\sigma_{g}$ & 1,600 & 1,760 & $n_{r}$ & 1,836 & 1,836 \\
\hline & $r_{e f f}(\mu m)$ & $6,221 \cdot 10^{-1}$ & $5,656 \cdot 10^{-2}$ & $n_{i}$ & $4,400 \cdot 10^{-3}$ & $4,800 \cdot 10^{-3}$ \\
\hline \multirow[t]{3}{*}{$p r$} & $r_{g}(\mu m)$ & $3,583 \cdot 10^{-1}$ & $2,544 \cdot 10^{-2}$ & & & \\
\hline & $\sigma_{g}$ & 2,000 & 2,200 & $n_{r}$ & 1,836 & 1,836 \\
\hline & $r_{e f f}(\mu m)$ & 1,061 & $1,204 \cdot 10^{-1}$ & $n_{i}$ & $5,500 \cdot 10^{-3}$ & $6,000 \cdot 10^{-3}$ \\
\hline \multirow[t]{3}{*}{$p p$} & $r_{g}(\mu m)$ & $3,583 \cdot 10^{-1}$ & $2,544 \cdot 10^{-2}$ & & & \\
\hline & $\sigma_{g}$ & 2,400 & 2,640 & $n_{r}$ & 1,836 & 1,836 \\
\hline & $r_{e f f}(\mu m)$ & 1,406 & $2,650 \cdot 10^{-1}$ & $n_{i}$ & $6,600 \cdot 10^{-3}$ & $7,200 \cdot 10^{-3}$ \\
\hline Mixing state cases & Parameters & Black Carbon & Sulfate & Parameters & Black Carbon & Sulfate \\
\hline \multirow[t]{3}{*}{ em } & $r_{g}^{*}(\mu m)$ & $1,180 \cdot 10^{-2}$ & $6,950 \cdot 10^{-2}$ & & & \\
\hline & $\sigma_{g}^{*}$ & 2,000 & 2,030 & $n_{r}^{*}$ & 1,750 & 1,430 \\
\hline & $r_{e f f}^{*}(\mu m)$ & $3,922 \cdot 10^{-2}$ & $2,433 \cdot 10^{-1}$ & $n_{i}^{*}$ & $4,400 \cdot 10^{-1}$ & $1,000 \cdot 10^{-8}$ \\
\hline \multirow[t]{3}{*}{ im } & $r_{g}(\mu m)$ & $1,664 \cdot 10^{-2}$ & & & & \\
\hline & $\sigma_{g}$ & 2,000 & & $n_{r}$ & 1,544 & \\
\hline & $r_{e f f}(\mu m)$ & $5,530 \cdot 10^{-2}$ & & $n_{i}$ & $1,570 \cdot 10^{-1}$ & \\
\hline
\end{tabular}

providing as inputs all the microphysical properties defined in Section 3.1. The calculations have been performed at all the wavelengths of the OPAC spectrum (at which the refractive indices were available) covering the spectral bands of the RRTMG_SW. Following Liao and Seinfeld (1998), we then derived the band optical properties by averaging the spectral optical properties within the RRTMG_SW spectral bands using the solar flux $\left(S_{\lambda}\right)$ as weight function at each wavelength:

$$
\begin{aligned}
Q_{b}^{\text {ext }} & =\frac{\sum_{\lambda} Q_{\lambda}^{\text {ext }} S_{\lambda}}{\sum_{\lambda} S_{\lambda}}, \\
\omega_{b} & =\frac{\sum_{\lambda} \omega_{\lambda} S_{\lambda}}{\sum_{\lambda} S_{\lambda}}, \\
g_{b} & =\frac{\sum_{\lambda} g_{\lambda} S_{\lambda}}{\sum_{\lambda} S_{\lambda}},
\end{aligned}
$$

in which the sums are defined over all the wavelengths falling within each RRTMG_SW spectral band (indexed by $b$ ).

Once the band optical properties have been calculated, we derived the correspondent layer radiative properties to be given as inputs to the RRTMG_SW, namely layer optical depth $\left(\tau_{b}\right)$, single scattering albedo and asymmetry factor, for all the species. For single 
and internally mixed species, layer single scattering albedo and asymmetry factor (intensive properties) coincide with the correspondent band optical properties above defined while the layer optical depth has been calculated with the following formula (Tegen \& Lacis, 1996):

$$
\tau_{b}=\frac{3 Q_{b}^{e x t}}{4 \rho r_{e f f}} M \Delta Z,
$$

in which, in addition to the already presented symbols, $\Delta Z>0$ is the atmospheric layer thickness. The layer radiative properties of the externally mixed species have been calculated starting from that of black carbon and sulfate (using the same weighted means of Eqs. (1)), as follows:

$$
\begin{aligned}
\tau_{b} & =\tau_{b, b c}+\tau_{b, s u}, \\
\omega_{b} \tau_{b} & =\omega_{b, b c} \tau_{b, b c}+\omega_{b, s u} \tau_{b, s u}, \\
g_{b} \omega_{b} \tau_{b} & =g_{b, b c} \omega_{b, b c} \tau_{b, b c}+g_{b, s u} \omega_{b, s u} \tau_{b, s u} .
\end{aligned}
$$

Eq. (11) for layer optical depth obviously requires assumptions on layer thickness and mass concentration. Following Liao and Seinfeld (1998), we supposed the aerosols located in two idealized homogeneous layers, with different heights and thicknesses, one for mineral dust and one for the anthropogenic species. This configuration with idealized layers has been tested against a more complex and realistic configuration with aerosol profiles covering several layers: in the context of our analysis the two configurations have been found to give substantially the same results and so the more simple configuration with idealized layers has been preferred. Within the layers, we then set three increasing mass concentrations for each species, aiming to simulate aerosol events of increasing intensity: weak, strong and extreme. In literature there is no well defined methodology to identify the strength of aerosol events but many researchers have used the aerosol optical depth (good measure of the aerosol load in the entire atmospheric column) for this purpose (Gkikas, Hatzianastassiou, \& Mihalopoulos, 2009). Following this approach, mass concentrations able to produce opportune layer optical depths defining the three aerosol events have been set. Only optical depths relative to the $4^{\text {th }}$ spectral band $(0,442-0,625 \mu \mathrm{m})$ have been used to define the events because this band includes the central wavelength of the visible range $(0,550 \mu \mathrm{m})$, usually considered for aerosol optical depth model estimate comparisons (Kinne et al., 2006). However, the spectral variability of the optical depth, not used for the event definition, has been considered in the optical analysis. The extreme event values have been selected in order to simulate very intense outbreaks registered over the Mediterranean basin for mineral dust $\left(\tau_{4}=3,0\right)$ (Cachorro et al., 2008; Pérez, Nickovic, Pejanovic, Baldasano, \& Özsoy, 2006), and heavily polluted urban areas (as for example Chinese mega cities) for the anthropogenic aerosols $\left(\tau_{4}=1,0\right)$ (Chan \& Yao, 2008; Xia et al., 2006). The strong event values, simply defined as half of the extreme values, have been selected to simulate important dust outbreaks in the Mediterranean and moderately polluted urban areas, respectively. Finally, the weak event values aimed to approximately simulate events of ordinary intensity, as for example could be produced by the climatological aerosol background far from sources $\left(\tau_{4}=0,1\right.$ for all the species). In Table 3 layer thicknesses, mass concentrations and corresponding layer optical depths defining the three events for all the species considered are reported. It is important to note that for mineral dust and organic carbon this event definition refers to the reference species. In the perturbed simulations the mass concentrations are conserved while the layer optical depths change according to the variability of optical and microphysical properties. Moreover, in the case of the black carbon-sulfate mixture, the three events have been defined separately for black carbon and sulfate: the conserved mass concentration of the mixture, for both external and internal mixing cases, is simply given by the sum of the two single species mass concentrations while the layer optical depth changes depending on the mixing state.

For all the species, we evaluated the particle microphysical perturbation impact on the layer radiative properties (generically indicated with $x$ ) through the relative spreads $(\Delta)$, that is to say the ratios of the perturbation-induced spreads of the mean layer radiative properties (maximum $\left\langle x>^{+}\right.$minus minimum $\langle x\rangle^{-}$) to the mean reference properties (marked with ${ }^{(*)}$ ):

$$
\Delta_{x}=\frac{\langle x\rangle^{+}-\langle x\rangle^{-}}{\left\langle x^{*}\right\rangle} .
$$

All the means have been calculated over the RRTMG_SW spectral bands using the band solar flux as weight function. Note that the bands $3 \leq b \leq 6$ (approximately visible-NIR bands, see Table 1) contain more than $75 \%$ of the total flux. The definition used for the

\begin{tabular}{|c|c|c|c|c|c|}
\hline $\begin{array}{l}\text { Parameters } \\
\Delta z(m)\end{array}$ & $\begin{array}{l}\text { Aerosol Events } \\
\text { - }\end{array}$ & $\begin{array}{l}\text { Mineral Dust } \\
3700-4500\end{array}$ & $\begin{array}{l}\text { Organic Carbon } \\
0-1100\end{array}$ & $\begin{array}{l}\text { Black Carbon } \\
0-1100\end{array}$ & $\begin{array}{l}\text { Sulfate } \\
0-1100\end{array}$ \\
\hline \multirow[t]{3}{*}{$M\left(\mathrm{~kg} / \mathrm{m}^{3}\right)$} & weak & $1,472 \cdot 10^{-7}$ & $0,304 \cdot 10^{-7}$ & $0,092 \cdot 10^{-7}$ & $0,282 \cdot 10^{-7}$ \\
\hline & strong & $1,472 \cdot 15 \cdot 10^{-7}$ & $0,304 \cdot 5 \cdot 10^{-7}$ & $0,092 \cdot 5 \cdot 10^{-7}$ & $0,282 \cdot 5 \cdot 10^{-7}$ \\
\hline & extreme & $1,472 \cdot 30 \cdot 10^{-7}$ & $0,304 \cdot 10 \cdot 10^{-7}$ & $0,092 \cdot 10 \cdot 10^{-7}$ & $0,282 \cdot 10 \cdot 10^{-7}$ \\
\hline \multirow[t]{3}{*}{$\tau_{4}$} & weak & 0,1 & 0,1 & 0,1 & 0,1 \\
\hline & strong & 1,5 & 0,5 & 0,5 & 0,5 \\
\hline & extreme & 3,0 & 1,0 & 1,0 & 1,0 \\
\hline
\end{tabular}
relative spreads has been found to be the most appropriate in terms of quantitative correlations to describe the effective impact of the

Table 3

Layer thicknesses $(\Delta z)$, mass concentrations $(M)$ and corresponding layer optical depths $\left(\tau_{4}\right)$, relative to the $4^{\text {th }}$ spectral band $(0,442-0$, $625 \mu m)$, set to define the three aerosol events for mineral dust, organic carbon and the black carbon-sulfate mixture. 
perturbed layer radiative properties on the direct radiative effect simulations (among other tested definitions). Note that the relative spreads in general do not depend on the aerosol event intensity, since the optical depth is directly proportional to the mass concentration (the same for reference and perturbed layer radiative properties) and single scattering albedo and asymmetry factor are intensive properties. For the black carbon-sulfate mixture, the relative spreads between the internal mixing case (perturbation case) and the external mixing case (reference case) have been calculated.

\subsection{Aerosol direct radiative effects}

The RRTMG_SW has been configured to simulate radiative fluxes during summer, at mid-latitudes and over land. For this purpose, we set a "Standard Mid-Latitude Summer Atmosphere" structure (with 51 vertical layers) as input file for the code and the summer solstice day (21 of June) as simulation date (this affects the scaling of the solar flux depending on the Sun-Earth distance). The radiative fluxes, and consequently the direct radiative effects, are highly sensitive to the geophysical context selected for the simulations (mainly to the $S Z A$ ). For this reason, in order to provide results suitable for general atmospheric scenarios, in this work we analyzed only relative variations of the direct radiative effects, meaning variations always evaluated against base net radiative fluxes (simulated in absence of aerosols, see below). However, the $S Z A$ has been found to have a relevant impact also on the relative variations of the direct radiative effects. Hence, we performed simulations for four different $S Z A\left(20^{\circ}, 40^{\circ}, 60^{\circ}\right.$ and $\left.80^{\circ}\right)$ therefore providing results ranging between extreme cases (not always coinciding with extreme $S Z A$ values). For each $S Z A$ we set a different surface albedo, following Briegleb, Minnis, Ramanathan, and Harrison (1986). At first, we selected four surface types suitable for mid-latitude lands, each one composed of a primary (I) and a secondary (II) component with different relative contributions (percentages within parentheses):

1. I: cultivated ground (80\%) - II: tall grassland (20\%);

2. I: grassland (80\%) - II: shrubland $(20 \%)$;

3. I: shrubland (80\%) - II: grassland (20\%);

4. I: woodland (80\%) - II: grassland (20\%).

Then, after averaging the broadband albedo values for the four surface types reported in Briegleb, Minnis, Ramanathan, and Harrison (1986) (spectrally integrated albedos weighted by the solar flux: considering broadband albedos instead of spectrally variable albedos has been found to have a negligible impact of $\lesssim 5 \%$ on our results), we derived the albedos $(A)$ for different $S Z A$ with the following formula (the already defined $\mu_{s}$ is the cosine of the $S Z A$ ) (Briegleb, Minnis, Ramanathan, \& Harrison, 1986):

$$
A\left(\mu_{s}\right)=A_{60} \frac{(1+d)}{\left(1+2 d \mu_{s}\right)}
$$

where $A_{60}$ is the reference surface albedo at $S Z A=60^{\circ}$ and $d$ is an empirical correction factor $(d=0,4$ for all the surface types selected (Briegleb, Minnis, Ramanathan, \& Harrison, 1986)). The surface albedos used in this work (derived through the described procedure) are reported in Table 4. Moreover, the surface has been assumed Lambertian (isotropic reflected radiation) and the clearsky option has been selected (no cloud layers) for all the simulations. The aerosol layers have been activated separately for mineral dust, organic carbon and the black carbon-sulfate mixture with all the properties and assumptions described in Sections 3.1 and 3.2.

From the RRTMG_SW outputs, we used the integrated (summed over all the spectral bands) net radiative fluxes at the TOA $\left(F_{t o a}=F_{\text {toa }}^{\downarrow}-F_{\text {toa }}^{\uparrow}\right)$ and at SFC $\left(F_{s f c}=F_{s f c}^{\downarrow}-F_{s f c}^{\uparrow}\right)$ to calculate the short-wave direct radiative effects $(E)$ as the instantaneous net flux variations arising from activating and deactivating the aerosol layers ( $a$ and 0 mean with and without aerosols, respectively):

$$
\begin{aligned}
E_{t o a} & =F_{t o a}^{a}-F_{t o a}^{0}=F_{t o a}^{\uparrow 0}-F_{t o a}^{\uparrow a}, \\
E_{s f c} & =F_{s f c}^{a}-F_{s f c}^{0} .
\end{aligned}
$$

Negative direct radiative effects indicate higher upward fluxes at the TOA and lower downward fluxes at SFC with aerosols, hence cooling in both cases and vice versa. Always in this work "lower" and "higher" direct radiative effects refer to their intensity, independently of their sign. Hence, negative and positive effects increase when they become more negative and more positive, respectively, and vice versa. For each aerosol species, we then calculated the relative variability ranges of the direct radiative effects

Table 4

Broadband surface albedos $(A)$ for all the solar zenith angles ( $S Z A)$ set for the radiative flux simulations (from Briegleb, Minnis, Ramanathan, and Harrison (1986)).

\begin{tabular}{ll}
\hline$S Z A$ & $A(\%)$ \\
\hline $20^{\circ}$ & 15,1 \\
$40^{\circ}$ & 16,4 \\
$60^{\circ}$ & 18,9 \\
$80^{\circ}$ & 23,3 \\
\hline
\end{tabular}


$(\Gamma)$, at the TOA and at SFC, as the ratios of their perturbation-induced variability ranges (maximum $E^{+}$minus minimum $E^{-}$: taken always positive) to the net fluxes in absence of aerosols:

$$
\begin{gathered}
\Gamma_{t o a}=\frac{\left|E_{t o a}^{+}-E_{t o a}^{-}\right|}{F_{t o a}^{0}}, \\
\Gamma_{s f c}=\frac{\left|E_{s f c}^{+}-E_{s f c}^{-}\right|}{F_{s f c}^{0}} .
\end{gathered}
$$

This definition has been adopted in order to evaluate the perturbation-induced variability ranges taking into account the geophysical context in which the direct radiative effects are simulated (measured by the base net fluxes simulated in absence of aerosols) and so to provide results suitable for general atmospheric scenarios. For the black carbon-sulfate mixture, the relative variability ranges produced by considering internal mixing (perturbation case) or external mixing (reference case) have been calculated.

\section{Results}

We carried out the result analysis at two levels: (i) impact of particle microphysical perturbations on layer radiative properties $(\Delta)$ and (ii) consequent impact on direct radiative effects $(\Gamma)$. In this section the results are presented separately for mineral dust, organic carbon and the black carbon-sulfate mixture. Note that only the figures of direct radiative effects for $S Z A=60^{\circ}$ are shown (the results for all the $S Z A$ are presented in the tables).

\subsection{Mineral dust}

The size distribution perturbations have a remarkable impact on $\tau_{b}\left(\Delta_{\tau}=152,3 \%\right)$ and negligible impacts on $g_{b}$ and $\omega_{b}\left(\Delta_{g}=9,8 \%\right.$; $\Delta_{\omega}=7,5 \%$ ). As shown in Fig. 1-a, increasing the particle size $\left(r_{\text {eff }}\right)$ causes the spectral maximum of $\tau_{b}$ (scattering maximum (Hansen \& Travis, 1974): for reference mineral dust occurring for $0,778<\lambda<1,242 \mu \mathrm{m}$ ) to shift towards longer wavelengths and to decrease in intensity (due to its inverse dependence on $r_{\text {eff }}$ : see Eq. (11)). The opposite occurs for decreasing $r_{\text {eff }}$ (this obvious statement is omitted for the other perturbed microphysical parameters and layer radiative properties in the rest of the current analysis). Consequently, in more than half of the short-wave region considered (approximately for $\lambda<2,151 \mu \mathrm{m}) \tau_{b}$ decreases with increasing $r_{\text {eff }}$ while the opposite occurs in the other bands. In all the dominant spectral bands $(3 \leq b \leq 6 \rightarrow 0,345<\lambda<1,242 \mu$ m: bands containing more than $75 \%$ of the solar flux) $\tau_{b}$ is very highly perturbed: the highest absolute spreads are $\sim(0,20 ; 3,03 ; 6,07)$ (between $\mathrm{pp}$ and $\mathrm{mm}$, for $0,442<\lambda<0,625 \mu \mathrm{m}$ ) in weak, strong and extreme events, respectively. Due to the shift of the scattering spectral maximum, $\omega_{b}$ (Fig. 1-b) decreases in the first bands and increases in the last bands with increasing $r_{\text {eff }}$ (more evidently for $\lambda<0,625$ and for $\lambda>2,500 \mu \mathrm{m}$, respectively, where the absorption of the reference dust is important with respect to the scattering).
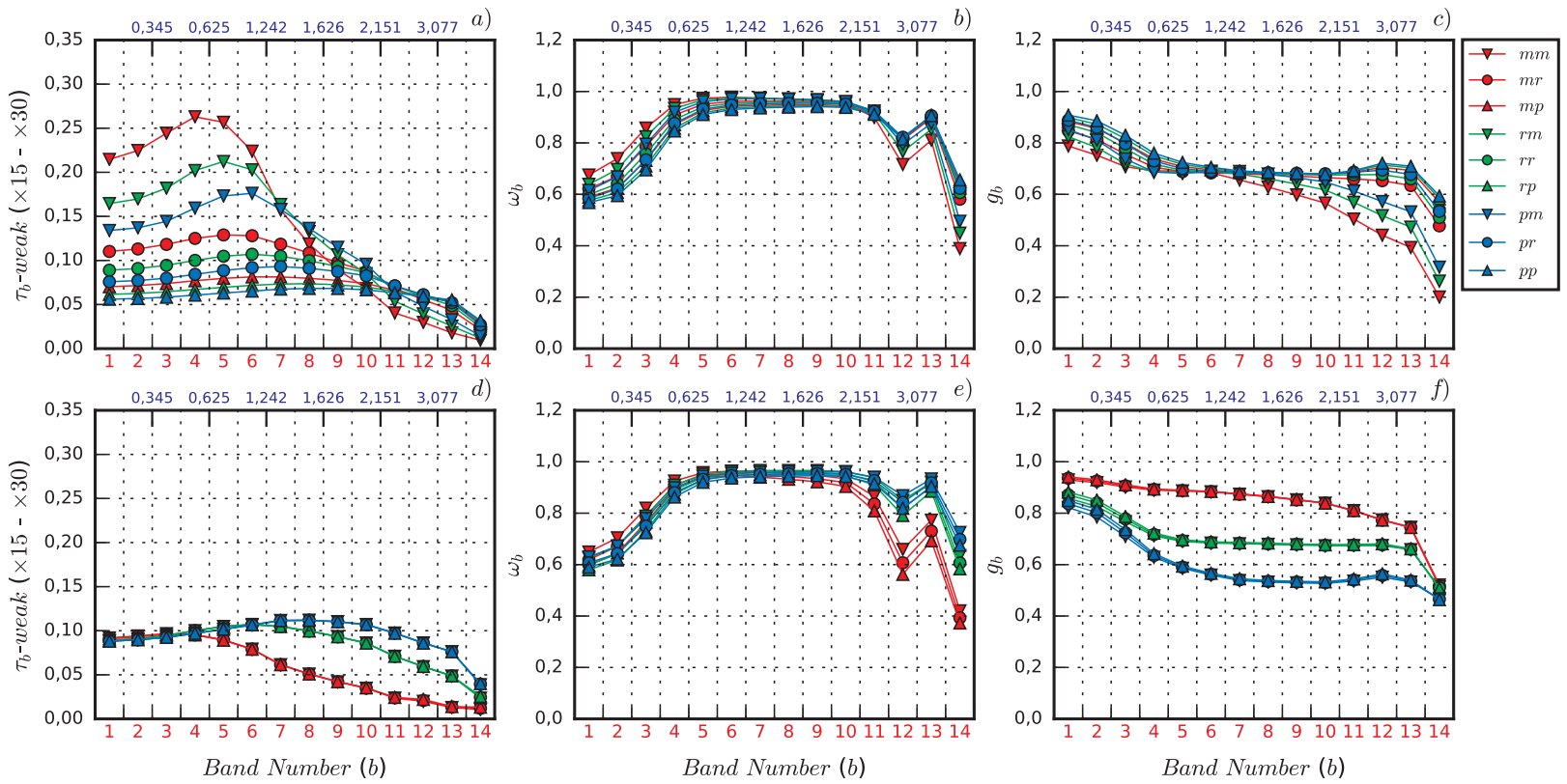

Fig. 1. Layer optical depth $\left(\tau_{b}\right)$, single scattering albedo $\left(\omega_{b}\right)$ and asymmetry factor $\left(g_{b}\right)$, in function of the spectral bands $(b)$, produced by perturbations of size distribution $(a, b, c)$ and refractive index $(d, e, f)$ for mineral dust. The optical depth is plotted only in the weak event, since it is enough to scale the $y$-axis values (by the factors within parentheses in the $y$-axis label) to obtain the values in strong and extreme events, respectively. The wavelength values reported at the top of the subplots (in $\mu \mathrm{m}$ ) represent division wavelengths between different spectral bands and are a subset of that for all the spectral bands reported in Table 1 . The perturbation case acronyms in the legend are explained in Section 3.1. 

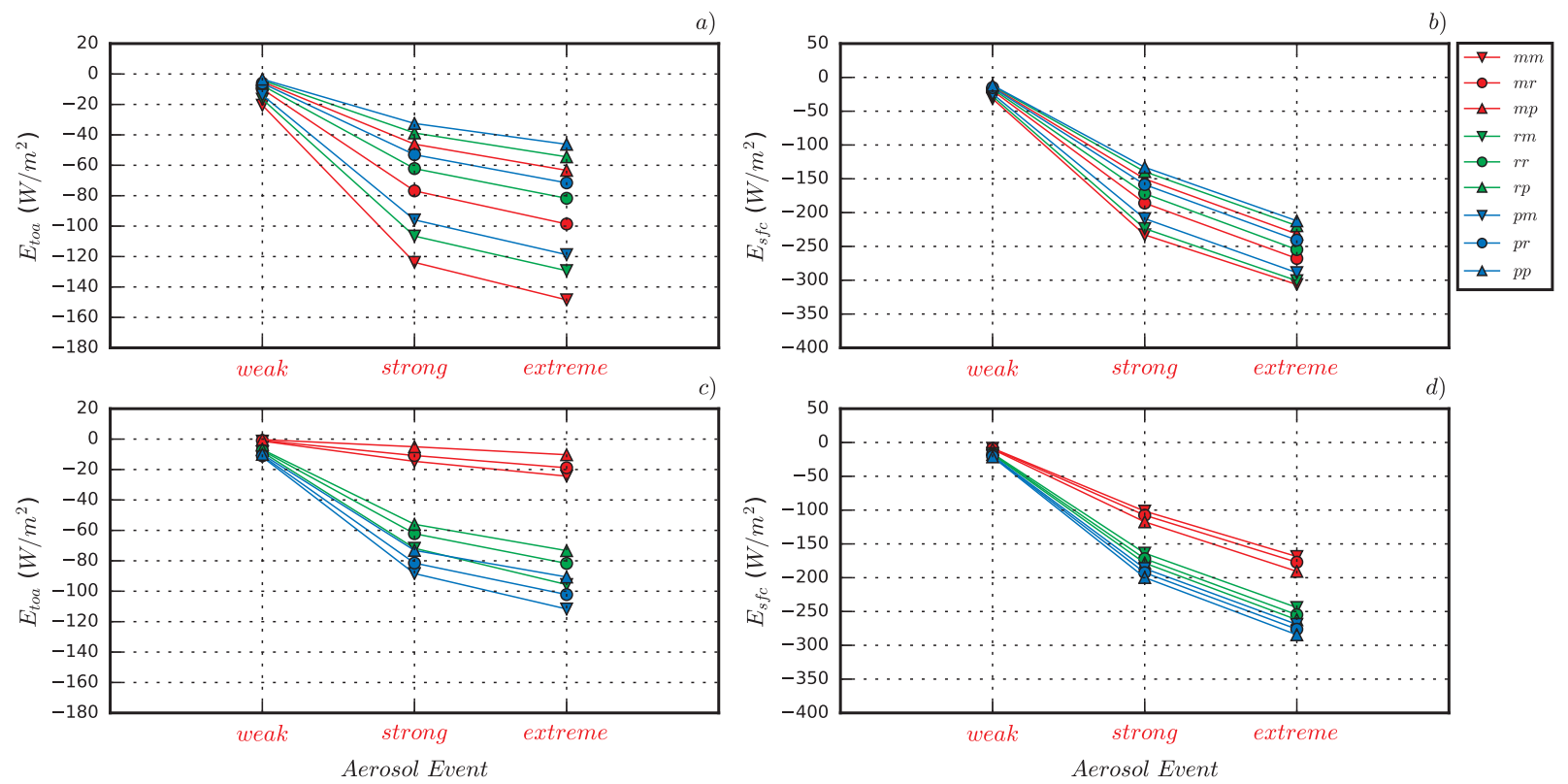

Fig. 2. Direct radiative effects $(E)$ at the TOA and at SFC, in the three events (for $S Z A=60^{\circ}$ ), produced by perturbations of size distribution $(a, b)$ and refractive index $(c, d)$ for mineral dust. The perturbation case acronyms in the legend are explained in Section 3.1.

However, in almost all the dominant bands $\omega_{b}$ is slightly affected by the perturbations: the highest absolute spread is $\sim 0,16$ (between $p p$ and $m m$, for $0,345<\lambda<0,442 \mu \mathrm{m}$ ). Finally, $g_{b}$ (Fig. 1-c) increases with increasing $r_{e f f}$ in almost all the bands (more evidently for $\lambda<0,625$ and $\lambda>1,299 \mu \mathrm{m}$ ) because larger particles scatter more in the forward direction. However, in almost all the dominant bands $g_{b}$ is nearly unaffected by the perturbations: the highest absolute spread is $\sim 0,12$ (between $\mathrm{mm}$ and $\mathrm{pp}$, for $0,345<\lambda<0,442 \mu \mathrm{m})$.

The direct radiative effects simulated under size distribution perturbations stay negative and increase in intensity with decreasing $r_{\text {eff }}$ coherently with the behavior of $\tau_{b}$ (with more sensitivity to $\sigma_{g}$ than to $r_{g}$ ), both at the TOA (Fig. 2-a) and at SFC (Fig. 2-b) and in all the three events (in agreement with Liao and Seinfeld (1998)). These features remain for all the $S Z A$ but for $S Z A=20^{\circ}$ the $r p$ and $p p$ cases produce very small positive direct radiative effects $\left(<1 \mathrm{~W} / \mathrm{m}^{2}\right)$ in the weak event. The perturbation-induced variability of the direct radiative effects can be remarkable, both at the TOA and at SFC: the highest absolute ranges are $\sim\left(17 ; 142 ; 189 \mathrm{~W} / \mathrm{m}^{2}\right)$ at the TOA (between $p p$ and $m m$ ) and $\sim\left(20 ; 222 ; 280 \mathrm{~W} / \mathrm{m}^{2}\right)$ at SFC (between $p p$ and $\mathrm{mm}$ ), in weak, strong and extreme events, respectively (considering all the $S Z A)$. The corresponding relative variability ranges (Table 5$)$ are in general larger at SFC $(\Gamma<33,9 \%)$ than at the TOA $(\Gamma<19,0 \%)$. Note that from their definition it is quite obvious that the relative variability ranges increase with the aerosol event intensity and so this information is omitted in the rest of this analysis.

The refractive index perturbations have significant impacts on $g_{b}$ and $\tau_{b}\left(\Delta_{g}=40,1 \% ; \Delta_{\tau}=22,0 \%\right)$ and a negligible impact on $\omega_{b}$ $\left(\Delta_{\omega}=3,8 \%\right)$. As shown in Fig. 1-d, increasing the real refractive index causes the spectral maximum of $\tau_{b}$ to shift towards longer wavelengths and to increase in intensity, while perturbing the imaginary refractive index does not have any significant impact on $\tau_{b}$ (more or less an enhanced absorption balances a reduced scattering and vice versa). Consequently, in the first bands (approximately for $\lambda<0,625 \mu \mathrm{m}) \tau_{b}$ remains almost unaffected by the perturbations while it increases with increasing $n_{r}$ in the other bands. Only in two dominant spectral bands $(b=5,6)$, therefore, $\tau_{b}$ is moderately perturbed: the highest absolute spreads are $\sim(0,03 ; 0,42 ; 0,84)$ (between $m p$ and $r m$, for $0,778<\lambda<1,242 \mu \mathrm{m}$ ) in weak, strong and extreme events, respectively. The shift of the scattering spectral maximum causes $\omega_{b}$ (Fig. 1-e) to decrease in the first bands and to increase in the last bands with increasing $n_{r}$ (more evidently for $\lambda<0,625$ and for $\lambda>1,942 \mu \mathrm{m}$, respectively), while a larger $n_{i}$ reduces $\omega_{b}$ in all the bands, as expected. However, in all the

Table 5

Relative variability ranges of direct radiative effects $(\Gamma)$ at the TOA and at SFC, in the three events, for size distribution and refractive index perturbations for mineral dust. The two values within parentheses indicate the $\Gamma$ variability due to the different $S Z A$ used for the radiative simulations (minimum and maximum of $\Gamma$ not necessarily corresponding to extreme $S Z A)$. The values of the base net fluxes in absence of aerosols for the four different $S Z A$ are (in $\left.W / m^{2}\right)$ : $(1067,972 ; 824,009)$, $(856,087 ; 645,481),(538,988 ; 384,598)$ and $(171,938 ; 101,287)$, at (TOA; SFC) and for $S Z A=20^{\circ}, 40^{\circ}, 60^{\circ}, 80^{\circ}$, respectively.

\begin{tabular}{|c|c|c|c|c|c|}
\hline \multicolumn{2}{|c|}{ Mineral Dust Perturbations: } & \multicolumn{2}{|c|}{ Size Distribution } & \multicolumn{2}{|c|}{ Refractive Index } \\
\hline Parameters & Aerosol Events & TOA & SFC & TOA & SFC \\
\hline \multirow[t]{3}{*}{$\Gamma(\%)$} & weak & $(1,0 ; 7,8)$ & $(2,2 ; 10,7)$ & $(0,8 ; 5,8)$ & $(1,6 ; 7,8)$ \\
\hline & strong & $(13,3 ; 16,9)$ & $(15,7 ; 27,7)$ & $(10,2 ; 16,0)$ & $(18,8 ; 25,5)$ \\
\hline & extreme & $(16,5 ; 19,0)$ & $(16,2 ; 33,9)$ & $(15,5 ; 18,8)$ & $(20,2 ; 33,0)$ \\
\hline
\end{tabular}




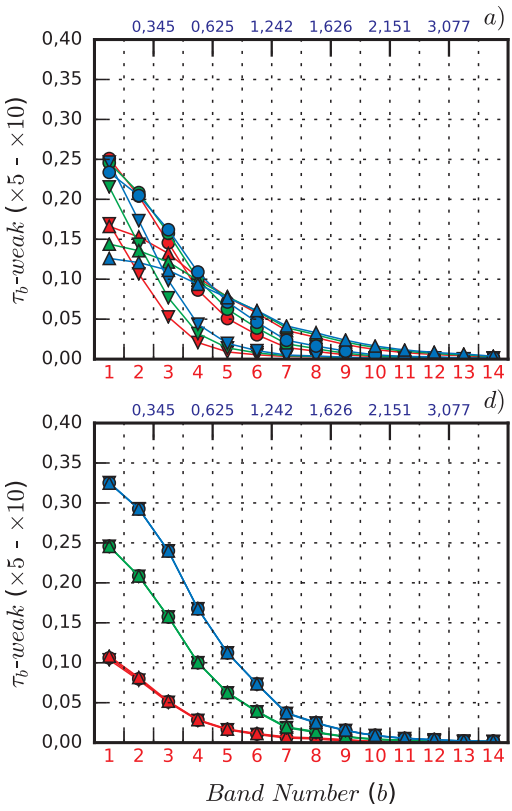

Band Number $(b)$
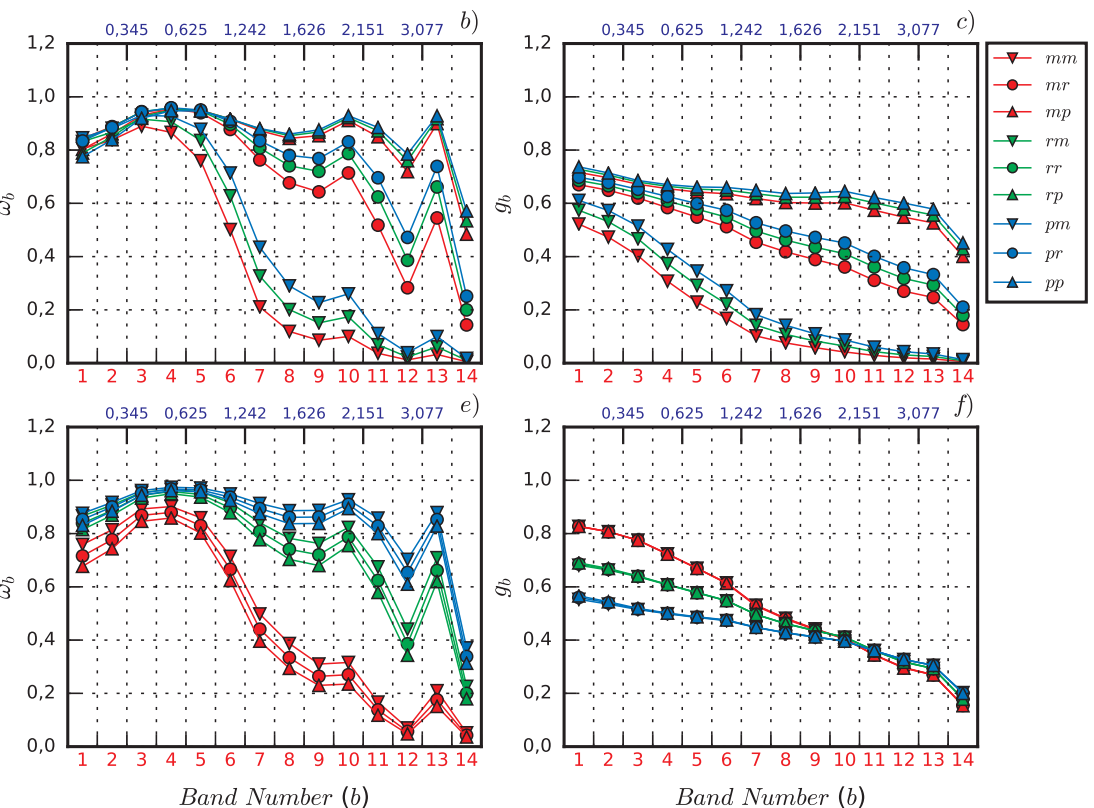

Band Number $(b)$

Fig. 3. Same as Fig. 1 but for organic carbon.

dominant bands $\omega_{b}$ is nearly unaffected by the perturbations: the highest absolute spread is $\sim 0$, 10 (between $p p$ and $m m$, for $0,345<\lambda<0,442 \mu \mathrm{m}$ ). Finally, $g_{b}$ (Fig. 1-f) decreases in all the bands with increasing $n_{r}$ (more evidently for $0,345<\lambda<3,846 \mu \mathrm{m}$ ) since a higher real part enhances reflection and refraction by the particles and consequently the backward scattering (Hansen \& Travis, 1974), while perturbing $n_{i}$ leaves unaffected $g_{b}$. In all the dominant bands $g_{b}$ is considerably affected by the perturbations: the highest absolute spread is of $\sim 0,33$ (between $\mathrm{pm}$ and $\mathrm{mp}$, for $0,778<\lambda<1,242 \mu \mathrm{m}$ ).

The direct radiative effects simulated under refractive index perturbations stay negative and increase in intensity with increasing $n_{r}$ and decreasing $n_{i}$ at the TOA (Fig. 2-c) and with increasing both $n_{r}$ and $n_{i}$ at SFC (Fig. 2-d) (with more sensitivity to $n_{r}$ than to $n_{i}$ ), in all the three events (in agreement with Liao and Seinfeld (1998) regarding $n_{i}$ ). These features remain for all the $S Z A$ but for $S Z A<60^{\circ}$ the simulations with reduced $n_{r}(m m, m r$ and $m p)$ produce in some cases positive direct radiative effects at the TOA (always preserving the same mutual quantitative relations). The perturbation-induced variability of the direct radiative effects again can be relevant, both at the TOA and at SFC: the highest absolute ranges are $\sim\left(11 ; 109 ; 165 \mathrm{~W} / \mathrm{m}^{2}\right.$ ) at the TOA (between $\mathrm{mp}$ and $\mathrm{pm}$ ) and $\sim\left(13 ; 173 ; 272 \mathrm{~W} / \mathrm{m}^{2}\right)$ at SFC (between $\mathrm{mm}$ and $\mathrm{pp}$ ), in weak, strong and extreme events, respectively (considering all the $S Z A$ ). The corresponding relative variability ranges (Table 5) are in general more extended at SFC $(\Gamma<33,0 \%)$ than at the TOA $(\Gamma<18,8 \%)$.

\subsection{Organic carbon}

The size distribution perturbations have significant impacts on $\tau_{b}, g_{b}$ and $\omega_{b}\left(\Delta_{\tau}=84,1 \% ; \Delta_{g}=78,9 \% ; \Delta_{\omega}=35,8 \%\right)$. As shown in Fig. 3-a, increasing the $r_{\text {eff }}$ causes the spectral maximum of $\tau_{b}$ to shift towards longer wavelengths and to decrease in intensity (as for mineral dust but with the scattering maximum of the reference species occurring at shorter wavelengths than those considered in this work). Consequently, in the first bands (approximately for $\lambda<0,625 \mu \mathrm{m}$ ) $\tau_{b}$ at first increases with increasing $r_{\text {eff }}$ but then starts to decrease (the maximum drop becomes dominant on its getting closer to the first bands), while it keeps increasing in the other bands. In all the dominant spectral bands $\tau_{b}$ is considerably perturbed: the highest absolute spreads are $\sim(0,11 ; 0,55 ; 1,09)$ (between $m m$ and $p r$, for $0,345<\lambda<0,442 \mu \mathrm{m})$ in weak, strong and extreme events, respectively. Due to the shift of the scattering spectral maximum, $\omega_{b}$ (Fig. 3-b) increases in almost all the bands with increasing $r_{\text {eff }}$ (more evidently for $\lambda>0$, $625 \mu m$, where the absorption is dominant with respect to the scattering by very small particles). However, only in two dominant bands $(b=5,6) \omega_{b}$ is strongly affected by the perturbations: the highest absolute spread is $\sim 0,42$ (between $\mathrm{mm}$ and $r p$, for $0,778<\lambda<1,242 \mu \mathrm{m}$ ). Finally, $g_{b}$ (Fig. 3-c) increases in all the bands with increasing $r_{e f f}$, as expected. In all the dominant bands $g_{b}$ is significantly affected by the perturbations: the highest absolute spread is $\sim 0,49$ (between $\mathrm{mm}$ and $\mathrm{pp}$, for $0,778<\lambda<1,242 \mu \mathrm{m}$ ).

The direct radiative effects simulated under size distribution perturbations stay negative and at first increase in intensity with increasing $r_{\text {eff }}$ starting then to decrease following the same behavior of $\tau_{b}$, both at the TOA (Fig. 4-a) and at SFC (Fig. 4-b), in all the three events. These features remain for all the $S Z A$ but for $S Z A=80^{\circ}$ the $m p$ case generates the most intense negative direct radiative effect at SFC instead of $p r$. The perturbation-induced variability of the direct radiative effects is moderate, both at the TOA and at SFC: the highest absolute ranges are $\sim\left(6 ; 24 ; 38 \mathrm{~W} / \mathrm{m}^{2}\right)$ at the TOA (between $\mathrm{mm}$ and $\mathrm{pr}$ ) and $\sim\left(8 ; 35 ; 65 \mathrm{~W} / \mathrm{m}^{2}\right)$ at SFC (between $\mathrm{mm}$ and $\mathrm{pr}$ ), in weak, strong and extreme events, respectively (considering all the $S Z A$ ). The corresponding relative variability ranges (Table 6) are in general more important at SFC $(\Gamma<20,3 \%)$ than at the TOA $(\Gamma<10,8 \%)$. 


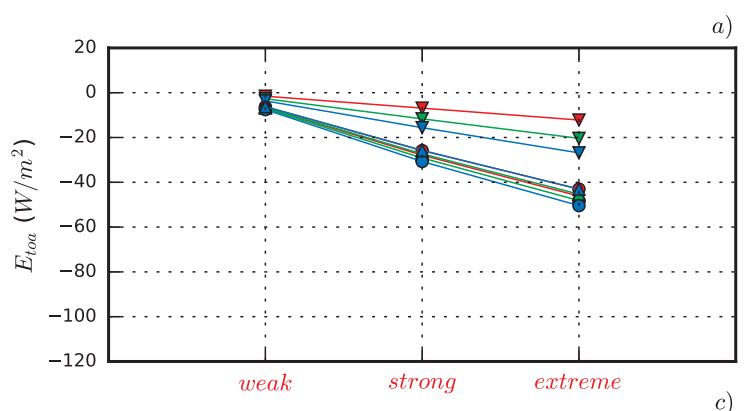

a)
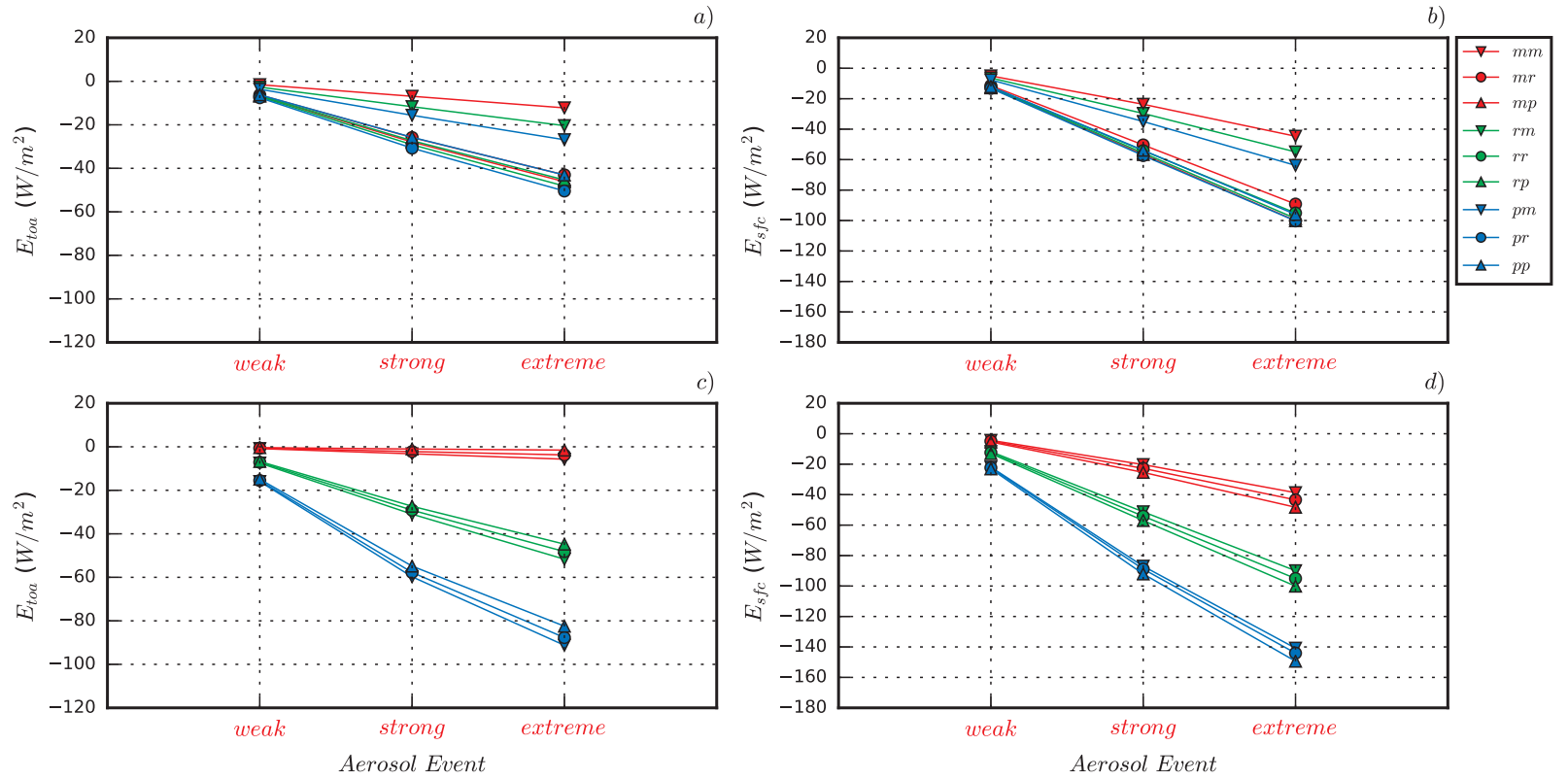

Fig. 4. Same as Fig. 2 but for organic carbon.

Table 6

Same as Table 5 but for organic carbon.

\begin{tabular}{|c|c|c|c|c|c|}
\hline \multicolumn{2}{|c|}{ Organic Carbon Perturbations: } & \multicolumn{2}{|c|}{ Size Distribution } & \multicolumn{2}{|c|}{ Refractive Index } \\
\hline Parameters & Aerosol Events & TOA & SFC & TOA & SFC \\
\hline \multirow[t]{3}{*}{$\Gamma(\%)$} & weak & $(0,3 ; 2,9)$ & $(0,7 ; 5,9)$ & $(1,3 ; 6,0)$ & $(2,2 ; 11,0)$ \\
\hline & strong & $(1,6 ; 8,5)$ & $(3,9 ; 16,9)$ & $(6,0 ; 15,4)$ & $(10,9 ; 26,2)$ \\
\hline & extreme & $(3,1 ; 10,8)$ & $(7,9 ; 20,3)$ & $(10,9 ; 19,3)$ & $(20,4 ; 30,1)$ \\
\hline
\end{tabular}

The refractive index perturbations have a remarkable impact on $\tau_{b}\left(\Delta_{\tau}=137,7 \%\right)$ and significant impacts on $\omega_{b}$ and $g_{b}$ $\left(\Delta_{\omega}=32,1 \% ; \Delta_{g}=29,3 \%\right.$ ). As shown in Fig. 3-d, increasing the real refractive index causes the spectral maximum of $\tau_{b}$ to shift towards longer wavelengths and to increase in intensity, while perturbing the imaginary refractive index does not show any significant impact on $\tau_{b}$. Consequently, in almost all the bands (more evidently for $\lambda<1,626 \mu \mathrm{m}$ ) $\tau_{b}$ increases with increasing $n_{r}$. In all the dominant spectral bands $\tau_{b}$ is very highly perturbed: the highest absolute spreads are $\sim(0,19 ; 0,95 ; 1,90)$ (between $\mathrm{mm}$ and $\mathrm{pm}$, for $0,345<\lambda<0,442 \mu \mathrm{m})$ in weak, strong and extreme events, respectively. The shift of the scattering spectral maximum causes $\omega_{b}$ (Fig. 3-e) to increase in all the bands with increasing $n_{r}$ (more evidently for $\lambda>0,625 \mu \mathrm{m}$ as for the increasing $r_{\text {eff }}$ ), while a larger $n_{i}$ reduces $\omega_{b}$ in all the bands, as expected (with a minor impact than that of $n_{r}$ ). Only in two dominant bands $(b=5,6)$, however, $\omega_{b}$ is strongly affected by the perturbations: the highest absolute spread is $\sim 0,32$ (between $m p$ and $p m$, for $0,778<\lambda<1,242 \mu \mathrm{m}$ ). Finally, $g_{b}$ (Fig. 3-f) decreases in almost all the bands with increasing $n_{r}$ (more evidently for $\lambda<1,626 \mu m$ and except for $\lambda>2,151$ $\mu m$ : in the limit $2 \pi r_{e f f} / \lambda \rightarrow 0, g_{b}$ deviates more rapidly for larger values of $n_{r}$ from its zero value for Rayleigh scattering (Hansen \& Travis, 1974)), while perturbing $n_{i}$ has a negligible impact on $g_{b}$. In all the dominant bands $g_{b}$ is moderately affected by the perturbations: the highest absolute spread is of $\sim 0,26$ (between $\mathrm{pm}$ and $\mathrm{mm}$, for $0,345<\lambda<0,442 \mu \mathrm{m}$ ).

The direct radiative effects simulated under refractive index perturbations stay negative and increase in intensity with increasing $n_{r}$ and decreasing $n_{i}$ at the TOA (Fig. 4-c) and with increasing both $n_{r}$ and $n_{i}$ at SFC (Fig. 4-d) (with much more sensitivity to $n_{r}$ than to $n_{i}$ ), in all the three events. These features remain for all the $S Z A$ but for $S Z A<60^{\circ}$ the simulations with reduced $n_{r}$ produce in some cases positive direct radiative effects at the TOA. The perturbation-induced variability of the direct radiative effects can be considerable, both at the TOA and at SFC: the highest absolute ranges are $\sim\left(16 ; 65 ; 116 \mathrm{~W} / \mathrm{m}^{2}\right)$ at the TOA (between $m p$ and $p m$ ) and $\sim\left(19 ; 90 ; 168 \mathrm{~W} / \mathrm{m}^{2}\right)$ at SFC (between $\mathrm{mm}$ and $\mathrm{pp}$ ), in weak, strong and extreme events, respectively (considering all the $S Z A$ ). The corresponding relative variability ranges (Table 6$)$ are in general more important at SFC $(\Gamma<30,1 \%)$ than at the TOA $(\Gamma<19,3 \%)$.

\subsection{Black carbon-sulfate mixture}

The mixing state perturbation has relevant impacts on $\omega_{b}, g_{b}$ and $\tau_{b}\left(\Delta_{\omega}=49,2 \% ; \Delta_{g}=37,7 \% ; \Delta_{\tau}=18\right.$, 4\%). Changing the mixing state means perturbing both size distribution and refractive index and so it is very difficult to isolate the effects of these perturbations on the layer radiative properties. However, the internally mixed species seems to behave like a smaller and more absorbing species 

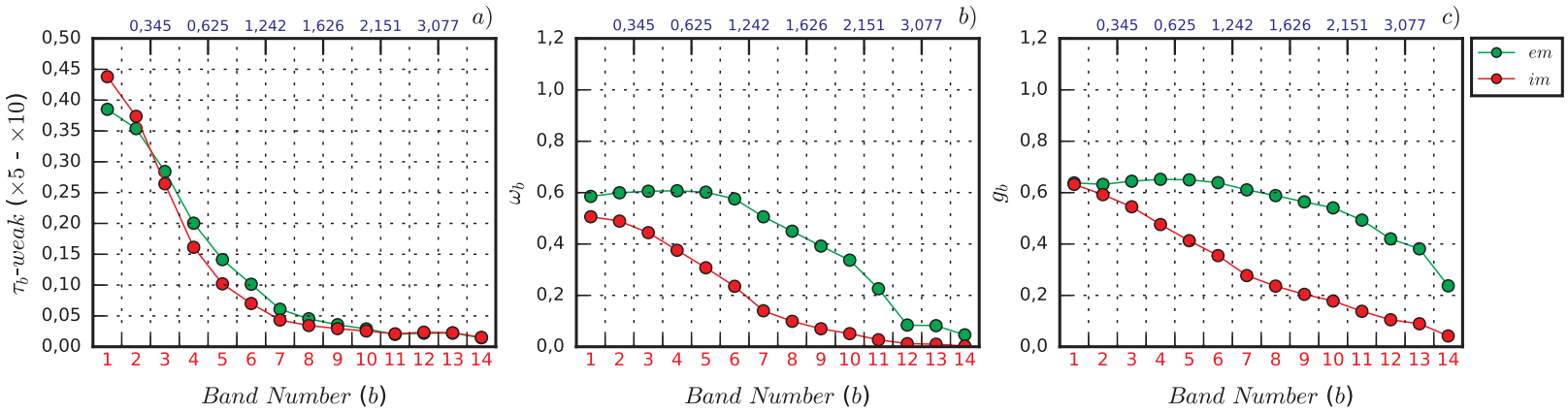

Fig. 5. Same as Fig. 1 but for the black carbon-sulfate mixture. Note that in this case only the mixing state is perturbed.

than the externally mixed species (regarding the scattering it is very difficult to infer information). As shown in Fig. 5-a, indeed, in the internal mixing case the spectral maximum of $\tau_{b}$ seems to shift towards shorter wavelengths and tends to grow. Consequently, $\tau_{b}$ in the internal mixing case is higher in the first two bands $(\lambda<0,345 \mu \mathrm{m})$ and slightly lower in the other bands (more evidently for $0,345<\lambda<1,942 \mu \mathrm{m}$ ) (in agreement with Lesins, Chylek, and Lohmann (2002)). In all the dominant spectral bands, therefore, $\tau_{b}$ is weakly perturbed: the highest absolute spreads between internal and external mixing cases are $\sim(0,04$; 0, 20; 0, 39) (for $0,625<\lambda<0,778 \mu \mathrm{m}$ ) in weak, strong and extreme events, respectively. The internally mixed species also has a lower $\omega_{b}$ (Fig. 5-b) in all the bands (more evidently for $0,263<\lambda<2,500 \mu \mathrm{m}$ ) (in agreement with Lesins, Chylek, and Lohmann (2002)). In all the dominant bands $\omega_{b}$ is strongly affected by the perturbations: the highest absolute spread between internal and external mixing cases is $\sim 0,34$ (for $0,778<\lambda<1,242 \mu \mathrm{m}$ ). Finally, also $g_{b}$ (Fig. 5-c) in the internal mixing case is lower in almost all the bands (approximately for $\lambda>0,345 \mu \mathrm{m}$ ). In all the dominant bands also $g_{b}$ is highly affected by the perturbations: the highest absolute spread between internal and external mixing cases is $\sim 0,28$ (for $0,778<\lambda<1,242 \mu \mathrm{m}$ ). Following the explanations given for mineral dust and organic carbon, the described variations of the layer radiative properties could be interpreted as results of the combined effects of decreasing the size and increasing the absorption of the particles.

The direct radiative effects simulated under different mixing states are always positive at the TOA (Fig. 6-a) (due to the strong absorption of black carbon) and negative at SFC (Fig. 6-b). The im case generates more intense direct radiative effects, that is more positive at the TOA and more negative at SFC. These features remain for all the $S Z A$. The perturbation-induced variability of the direct radiative effects is quite low, both at the TOA and at SFC: the highest absolute ranges are $\sim\left(7 ; 19 ; 22 \mathrm{~W} / \mathrm{m}^{2}\right)$ at the TOA and $\sim\left(12 ; 34 ; 31 \mathrm{~W} / \mathrm{m}^{2}\right)$ at $\mathrm{SFC}$, in weak, strong and extreme events, respectively (considering all the $\left.S Z A\right)$. The corresponding relative variability ranges (Table 7$)$ are slightly more important at SFC $(\Gamma<6,2 \%)$ than at the TOA $(\Gamma<5,7 \%)$.

\section{Discussion}

Regarding the impact of the particle microphysical perturbations on the layer radiative properties, a very different behavior between mineral dust and organic carbon has been observed. For mineral dust, $\tau_{b}$ is more sensitive to the size distribution while $g_{b}$ to the refractive index ( $\omega_{b}$ is weakly affected by both the properties). For organic carbon, instead, $\tau_{b}$ is more sensitive to the refractive index while $g_{b}$ to the size distribution ( $\omega_{b}$ is more or less equally affected by the two properties). These differences seem to be caused mainly by the very different mean sizes of the two species. The maximum of the aerosol scattering efficiency, indeed, approximately occurs for $\lambda \sim r_{\text {eff }}$ and changes its spectral position depending on the particle microphysical properties (both size distribution and refractive index) (Hansen \& Travis, 1974). Clearly the effects of the particle microphysical perturbations on the layer radiative properties strongly depend on the original spectral position of the scattering maximum (as can be inferred from Figs. 1 and 3). Even if the layer radiative properties of organic carbon seem to be more sensitive (higher relative spreads) than that of mineral dust to the particle microphysical properties (at least in the short-wave region considered in this work), note that the relative spreads of the two
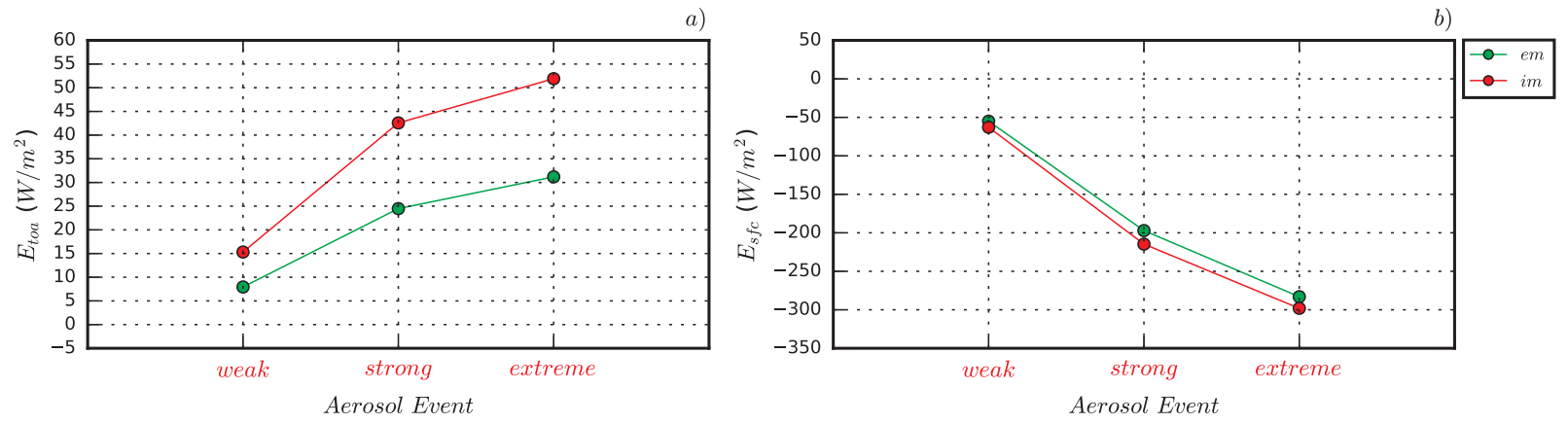

Fig. 6. Same as Fig. 2 but for the black carbon-sulfate mixture. Note that in this case only the mixing state is perturbed. 
Table 7

Same as Table 5 but for the black carbon-sulfate mixture.

\begin{tabular}{|c|c|c|c|}
\hline \multicolumn{2}{|c|}{ Black Carbon-Sulfate Mixture Perturbation: } & \multicolumn{2}{|c|}{ Mixing State } \\
\hline Parameters & Aerosol Events & TOA & SFC \\
\hline \multirow[t]{3}{*}{$\Gamma(\%)$} & weak & $(0,6 ; 2,9)$ & $(1,4 ; 3,0)$ \\
\hline & strong & $(1,7 ; 5,5)$ & $(4,1 ; 6,2)$ \\
\hline & extreme & $(1,9 ; 5,7)$ & $(3,8 ; 4,9)$ \\
\hline
\end{tabular}

aerosol species are not directly comparable, because even if generated by the same particle microphysical perturbations they refer to different reference layer radiative properties. Regarding the black carbon-sulfate mixture, $\omega_{b}$ and $g_{b}$ seem to be more sensitive than $\tau_{b}$ to the mixing state. The internal mixing between black carbon and sulfate reduces the number of particles in the layer (the sulfate particles disappear but the total mass is conserved) but makes the black carbon particles larger (higher geometric cross section) and with a slightly reduced imaginary refractive index (from $4,400 \cdot 10^{-1}$ it becomes $1,570 \cdot 10^{-1}$, at $\lambda=0,550 \mu \mathrm{m}$ ). This causes the total extinction to remain approximately the same, the total absorption to increase and the forward scattering to decrease.

Also the perturbation-induced variations of the direct radiative effects show different features for mineral dust and organic carbon, coherently with the perturbation impacts on the layer radiative properties. For mineral dust, despite the highly different impact on $\tau_{b}$, size distribution and refractive index perturbations produce more or less comparable relative variability ranges, both at the TOA and at SFC and in all the three events. This could be explained considering that the refractive index perturbations have a significant impact on $g_{b}$ in dominant spectral bands (in which also $\omega_{g}$ is very high). In general, an increasing $g_{b}$ causes the direct radiative effects to become less negative (or more positive) both at the TOA and at SFC. This causes the relative variability ranges produced by the refractive index perturbations to be enhanced since the simulations with increasing $\tau_{b}$ have decreasing $g_{b}$ (and vice versa) in the dominant bands. For organic carbon, instead, the refractive index perturbations produce higher relative variability ranges than the size distribution perturbations, both at the TOA and at SFC and in the three aerosol events. This is firstly caused by the higher impact on $\tau_{b}$ of the refractive index perturbations. However, also the impacts on $g_{b}$ seem to have again a role, given that the $g_{b}$ spreads are produced in dominant spectral bands (with also high $\omega_{b}$ values) for both size distribution and refractive index perturbations. As for mineral dust, indeed, the impact on $g_{b}$ of the refractive index perturbations enhances the relative variability ranges (decreasing $g_{b}$ for simulations with increasing $\tau_{b}$ ). The opposite occurs for the size distribution perturbations because now the simulations with increasing $\tau_{b}$ have also increasing $g_{b}$ and this limits the extents of the relative variability ranges. The $\omega_{b}$ spreads are more pronounced in not dominant spectral bands for both size distribution and refractive index perturbations and also have approximately the same features for the two properties. However, in general an increasing $\omega_{b}$ causes the direct radiative effects to become more negative (or less positive) at the TOA and less negative at SFC. Hence, in both cases the perturbation impact on $\omega_{b}$ causes the relative variability ranges to be slightly enhanced at the TOA and reduced at SFC (simulations with increasing $\tau_{b}$ have also increasing $\omega_{b}$ ). Regarding the black carbon-sulfate mixture, despite the lower $\tau_{b}$ in the dominant spectral bands, the internally mixed species generates more intense direct radiative effects both at the TOA (more positive) and at SFC (more negative). The strong impact of the mixing state on $\omega_{b}$, indeed, causes the internally mixed particles to be much more absorbing and so to overbalance the small $\tau_{b}$ reduction (the diffuse fluxes are strongly depleted). The relative variability ranges, however, remain small both at the TOA and at SFC and in all the three events. This is due to both the low impact of the mixing state on $\tau_{b}$ and to the balancing effect of the $\omega_{b}$ perturbation-induced variation. Note that at SFC the relative variability ranges in the extreme events are lower than in the strong event. This is probably due to the strong depletion of the diffuse fluxes caused by the very low $\omega_{b}$ of the mixture (due to the presence of black carbon), which in the extreme event limits the perturbation-induced variations of the direct radiative effects (above all at SFC).

\section{Conclusions}

In this work, we carried out a systematic and detailed study on the propagation of particle microphysical perturbations on the optical and radiative simulations usually required by the ARI implementation in weather and climate models. Our analysis has been carried out at two levels: (i) impact of particle microphysical perturbations on layer radiative properties and (ii) consequent impact on short-wave direct radiative effects, at the TOA and at SFC. We set a simple idealized experiment, carried out with numerical tools widely used in the atmosphere modeling community, in order to isolate and analyze the radiative sensitivity to the particle microphysical parameterization in weather and climate models.

We found consistent results with Liao and Seinfeld (1998) regarding the sign of the direct radiative effect variations induced by local perturbations of size distribution and imaginary refractive index of mineral dust, both at the TOA and at SFC. Moreover, we found results in agreement with Lesins, Chylek, and Lohmann (2002) regarding the variations of optical depth and single scattering albedo due to a different mixing state between black carbon and sulfate (in particular the enhanced absorption in the internal mixing case) but an opposite result regarding the asymmetry factor: Lesins, Chylek, and Lohmann (2002) found a higher value at $\lambda=0,550 \mu \mathrm{m}$ in the internal mixing case while in this work we found a lower value in almost all the spectral bands for the internally mixed species. This different result can be explained by the opposite assumptions made to parameterize the homogeneous internal mixing between the two species. Lesins, Chylek, and Lohmann (2002) considered, indeed, a coagulation of a smaller mode (black carbon) into a larger mode (sulfate), which caused the internally mixed species to be larger than both the single species (and so to 
have a higher $g$ at $\lambda=0,550 \mu \mathrm{m}$ than the externally mixed species). In this work, instead, we considered a condensation of the sulfate particles (larger species) on the black carbon particles (smaller species), which caused the internally mixed species to act as a smaller species than the externally mixed one (and so to have a lower $g_{b}$ in almost all the bands).

In general, we found that the size distribution perturbations can produce more important relative variability ranges of direct radiative effects (considering the highest values produced by the variable $S Z A$ ) for mineral dust than for organic carbon, while the refractive index perturbations produce almost comparable relative variability ranges for the two aerosol species, both at the TOA and at SFC and in all the three aerosol events. Changing the mixing state of the black carbon-sulfate mixture produces in general minor relative variability ranges. Looking at all the particle microphysical perturbations for all the aerosol species considered, we found the following relative variability ranges: $\Gamma<7,8 \%$ and $\Gamma<11,0 \%$ in the weak event, $\Gamma<16,9 \%$ and $\Gamma<27,7 \%$ in the strong event, $\Gamma<19,3 \%$ and $\Gamma<33,9 \%$ in the extreme event, at the TOA and at SFC, respectively. The highest relative variability range at the TOA $(\Gamma=19,3 \%)$ is produced by the refractive index perturbations of organic carbon while at SFC $(\Gamma=33,9 \%)$ by the size distribution perturbations of mineral dust, in the extreme event. However, the relative variability ranges never exceed the perturbation range applied to the particle microphysical properties $( \pm 20 \% \rightarrow 40 \%)$, not even in the extreme event. This result can be considered as a quantitative measure of the propagation of the particle microphysical perturbations on the optical and radiative simulations usually performed in weather and climate models: local perturbations within a certain range on the particle microphysical properties produce variability ranges of direct radiative effects always within the perturbation range applied, if evaluated against the base net radiative fluxes in absence of aerosols. In other words, small uncertainties on the aerosol microphysical parameterization propagate on the simulated direct radiative effects mainly with a loss of strength. To conclude, the perturbation-induced absolute variations of the direct radiative effects have been found to be large, above all in strong and extreme events, and so potentially capable of significantly affecting the meteorological variables in weather and climate simulations. Hence, this work confirms and highlights the importance of improving the aerosol microphysical parameterization in atmospheric models, in order to achieve better model assessments of the ARI radiative effects and therefore better meteorological forecasts and climate predictions.

\section{Acknowledgements}

We thank the developers of the T-Matrix and RRTMG_SW codes for making them freely available and Niti Mishra for the revision of the English text.

\section{Vitae}

Vincenzo Obiso studied Theoretical Physics at the University of Rome (Italy) and is a Ph.D. student at the Earth Sciences Department of the Barcelona Supercomputing Center (BSC-ES, Barcelona, Spain) since 2013. His research plan focuses on the implementation of the dynamic aerosol-radiation interaction within the NMMB-MONARCH, an online integrated meteorology-chemistry model under development at the BSC-ES, and on the evaluation of its impact on the meteorological forecasts.

Oriol Jorba has a Ph.D. in Environmental Engineering (2005). His research activities have included high resolution mesoscale meteorology, air quality and development of online meteorology-chemistry models. In 2005, he was enrolled as researcher at the Earth Sciences Department of the Barcelona Supercomputing Center (BSC-ES, Barcelona, Spain) and in 2008 moved to the Atmospheric Modeling Group Manager position. Now he is senior researcher of the Atmospheric Composition group. He held a research position at the University of California Irvine (USA) in 2011 and at the NASA Goddard Institute for Space Studies (USA) in 2013. He has co-authored more than 50 papers in international scientific journals and over 100 communications to international conferences.

\section{Funding sources}

This work has been funded by the Spanish Ministry of Economy and Competitiveness [grant: CGL2013-46736-R] and by the ACTRIS Research Infrastructure Project of the European Union's Horizon 2020 research and innovation programme [grant agreement: No. 654169]. Further support has been provided by the Severo Ochoa Program, awarded by the Spanish Government [grant: SEV-2011-00067]. Vincenzo Obiso is funded by the Spanish Ministry of Economy and Competitiveness ['FPI-SO' grant: SVP-2013067953].

\section{References}

Badia, A., Jorba, O., Voulgarakis, A., Dabdub, D., Pérez García-Pando, C., Hilboll, A., ... Janjic, Z. (2017). Description and evaluation of the Multiscale Online Nonhydrostatic AtmospheRe CHemistry model (NMMB-MONARCH) version 1.0: gas-phase chemistry at global scale. Geoscientific Model Development, 10, 609-638.

Barker, H.W., Pincus, R., \& Morcrette, J.-J. (2003). The Monte-Carlo Independent Column Approximation: Application within large-scale models. In Proceedings of the GCSS/ARM Workshop on the Representation of Cloud Systems in Large-Scale Models, Kananaskis, Alberta, Canada, $20-24$ May 2002.

Bauer, S. E., Menon, S., Koch, D., Bond, T. C., \& Tsigaridis, K. (2010). A global modeling study on carbonaceous aerosol microphysical characteristics and radiative effects. Atmospheric Chemistry and Physics, 10, 7439-7456.

Boucher, O., Randall, D., Artaxo, P., Bretherton, C., Feingold, G., Forster, P., ... Zhang, X. Y. (2013). Clouds and Aerosols. In T. F. Stocker, D. Qin, G.-K. Plattner, M. Tignor, S. K. Allen, \& J. Boschung, (Eds.). Climate Change 2013: The Physical Science Basis. Contribution of Working Group I to the Fifth Assessment Report of the Intergovernmental Panel on Climate Change. Cambridge, United Kingdom and New York, NY, USA: Cambridge University Press.

Briegleb, B. P., Minnis, P., Ramanathan, V., \& Harrison, E. (1986). Comparison of Regional Clear-Sky Albedos Inferred from Satellite Observations and Model Computations. Journal of Climate and Applied Meteorology, 25, 214-226. 
Cachorro, V. E., Toledano, C., Prats, N., Sorribas, M., Mogo, S., Berjón, A., ... De Frutos, A. M. (2008). The strongest desert dust intrusion mixed with smoke over the Iberian Peninsula registered with Sun photometry. Journal of Geophysical Research, 113, D14S04.

Chan, C. K., \& Yao, X. (2008). Air pollution in mega cities in China. Atmospheric Environment, 42, 1-42.

Chin, M., Ginoux, P., Kinne, S., Torres, O., Holben, B. N., Duncan, B. N., ... Nakajima, T. (2002). Tropospheric Aerosol Optical Thickness from the GOCART Model and Comparisons with Satellite and Sun Photometer Measurements. Journal of the Atmospheric Sciences, 59, 461-483.

Clough, S. A., \& Iacono, M. J. (1995). Line-by-line calculation of atmospheric fluxes and cooling rates: 2. Application to carbon dioxide, ozone, methane, nitrous oxide and the halocarbons. Journal of Geophysical Research, 100(D8), 16519-16535.

Clough, S. A., Iacono, M. J., \& Moncet, J.-L. (1992). Line-by-line calculation of atmospheric fluxes and cooling rates: Application to water vapor. Journal of Geophysical Research, 97(D14), 15761-15785.

Clough, S. A., Shephard, M. W., Mlawer, E. J., Delamere, J. S., Iacono, M. J., Cady-Pereira, K., ... Brown, P. D. (2005). Atmospheric radiative transfer modeling: a summary of the AER codes. Journal of Quantitative Spectroscopy and Radiative Transfer, 91, 233-244.

DeCarlo, P. F., Slowik, J. G., Worsnop, D. R., Davidovits, P., \& Jimenez, J. L. (2004). Particle Morphology and Density Characterization by Combined Mobility and Aerodynamic Diameter Measurements. Part 1: theory. Aerosol Science and Technology, 38, 1185-1205.

Di Tomaso, E., Schutgens, N. A. J., Jorba, O., \& Pérez García-Pando, C. (2017). Assimilation of MODIS Dark Target and Deep Blue observations in the dust aerosol component of NMMB-MONARCH version 1.0. Geoscientific Model Development, 10, 1107-1129.

Fu, Q., \& Liou, K. N. (1992). On the Correlated k-Distribution Method for Radiative Transfer in Nonhomogeneous Atmospheres. Journal of the Atmospheric Sciences, 49(22), 2139-2156.

Gkikas, A., Hatzianastassiou, N., \& Mihalopoulos, N. (2009). Aerosol events in the broader Mediterranean basin based on 7-year (2000-2007) MODIS C005 data. Annales Geophysicae, 27, 3509-3522.

Hansen, J. E., \& Travis, L. D. (1974). Light scattering in planetary atmospheres. Space Science Reviews, 16, 527-610.

Hess, M., Köpke, P., \& Schult, I. (1998). Optical Properties of Aerosols and Clouds The Software Package OPAC. Bulletin of the American Meteorological Society, 79(5), $831-844$.

Iacono, M. J., (2011). Application of Improved Radiation Modeling to General Circulation Models. Atmospheric and Environmental Research, Final Technical Report: $1-39$.

Iacono, M. J., Delamere, J. S., Mlawer, E. J., Shephard, M. W., Clough, S. A., \& Collins, W. D. (2008). Radiative forcing by long-lived greenhouse gases: Calculations with the AER radiative transfer models. Journal of Geophysical Research, 113, D13103.

Jacobson, M. Z. (2005). Fundamentals of Atmospheric Modeling. New York, USA: Cambridge University Press.

Jorba, O., Dabdub, D., Blaszczak-Boxe, C., Pérez, C., Janjic, Z., Baldasano, J. M., ... Gonçalves, M. (2012). Potential significance of photoexcited NO 2 on global air quality with the NMMB/BSC chemical transport model. Journal of Geophysical Research, 117, D13301.

Kinne, S., Schulz, M., Textor, C., Guibert, S., Balkanski, Y., Bauer, S. E., ... Tie, X. (2006). An AeroCom initial assessment - optical properties in aerosol component modules of global models. Atmospheric Chemistry and Physics, 6, 1815-1834.

Lesins, G., Chylek, P., \& Lohmann, U. (2002). A study of internal and external mixing scenarios and its effect on aerosol optical properties and direct radiative forcing. Journal of Geophysical Research, 107(D10) AAC5 1-AAC5 12.

Liao, H., \& Seinfeld, J. H. (1998). Radiative forcing by mineral dust aerosols: sensitivity to key variables. Journal of Geophysical Research, 103(D24), 31637-31645.

Liu, P. F., Abdelmalki, N., Hung, H.-M., Wang, Y., Brune, W. H., \& Martin, S. T. (2015). Ultraviolet and visible complex refractive indices of secondary organic material produced by photooxidation of the aromatic compounds toluene and m-xylene. Atmospheric Chemistry and Physics, 15, $1435-1446$.

Meador, W. E., \& Weaver, W. R. (1980). Two-Streams Approximations to Radiative Transfer in Planetary Atmospheres: a Unified Description of Existing Methods and a New Improvement. Journal of the Atmospheric Sciences, 37, 630-643.

Mishchenko, M. I., \& Travis, L. D. (1998). Capabilities and limitations of a current FORTRAN implementation of the T-matrix method for randomly oriented, rotationally symmetric scatterers. Journal of Quantitative Spectroscopy and Radiative Transfer, 60, 309-324.

Mishchenko, M. I., Travis, L. D., \& Lacis, A. A. (2002). Scattering, Absorption and Emission of Light by Small Particles. Cambridge, UK: Cambridge University Press.

Mishchenko, M. I., Videen, G., Babenko, V. A., Khlebtsov, N. G., \& Wriedt, T. (2004). T-matrix theory of electromagnetic scattering by particles and its applications: a comprehensive reference database. Journal of Quantitative Spectroscopy and Radiative Transfer, 88, 357-406.

Mlawer, E. J., Taubman, S. J., Brown, P. D., Iacono, M. J., \& Clough, S. A. (1997). Radiative transfer for inhomogeneous atmosphere: RRTM, a validated correlated-k model for the longwave. Journal of Geophysical Research, 102(D14), 16663-16682.

Myhre, G., Samset, B. H., Schulz, M., Balkanski, Y., Bauer, S., Berntsen, T. K., ... Zhou, C. (2013a). Radiative forcing of the direct aerosol effect from AeroCom Phase II simulations. Atmospheric Chemistry and Physics, 13, 1853-1877.

Myhre, G., Shindell, D., Bréon, F.-M., Collins, W., Fuglestvedt, J., Huang, J., ... Zhang, H. (2013b). Anthropogenic and Natural Radiative Forcing. In T. F. Stocker, D. Qin, G.-K. Plattner, M. Tignor, S. K. Allen, \& J. Boschung, (Eds.). Climate Change 2013: The Physical Science Basis. Contribution of Working Group I to the Fifth Assessment Report of the Intergovernmental Panel on Climate Change. Cambridge, United Kingdom and New York, NY, USA: Cambridge University Press.

Nemesure, S., Wagener, R., \& Schwartz, S. E. (1995). Direct shortwave forcing of climate by the anthropogenic sulfate aerosol: Sensitivity to particle size, composition and relative humidity. Journal of Geophysical Research, 100(D12), 26105-26116.

Nickovic, S., Kallos, G., Papadopoulos, A., \& Kakaliagou, O. (2001). A model for prediction of desert dust cycle in the atmosphere. Journal of Geophysical Research, 106(D16), 18113-18129.

Obiso, V., Pandolfi, M., Ealo, M., \& Jorba, O. (2017). Impact of aerosol microphysical properties on mass scattering cross sections. Journal of Aerosol Science, 112, $68-82$.

Oreopoulos, L., \& Barker, H. W. (1999). Accounting for subgrid-scale cloud variability in a multi-layer 1D solar radiative transfer algorithm. Quarterly Journal of the Royal Meteorological Society, 125, 301-330.

Pérez, C., Haustein, K., Janjic, Z., Jorba, O., Huneeus, N., Baldasano, J. M., ... Thomson, M. (2011). Atmospheric dust modeling from meso to global scales with the online NMMB/BSC-Dust model - Part 1: Model description, annual simulations and evaluation. Atmospheric Chemistry and Physics, $11,13001-13027$.

Pérez, C., Nickovic, S., Pejanovic, G., Baldasano, J. M., \& Özsoy, E. (2006). Interactive dust-radiation modeling: A step to improve weather forecasts. Journal of Geophysical Research, 111, D16206.

Rap, A., Scott, C. E., Spracklen, D. V., Bellouin, N., Forster, P. M., Carslaw, K. S., ... Mann, G. (2013). Natural aerosol direct and indirect radiative effects. Geophysical Research Letters, 40, 3297-3301.

Shettle, E. P., (1984). Optical and radiative properties of a desert aerosol model. In Fiocco, G., editor, IRS '84: Current Problems in Atmospheric Radiation: Proceedings of the International Radiation Symposium, Perugia, Italy, 21-28 August 1984, pages 74-77. A. Deepak Publishing, Hampton, Virginia, USA.

Sokolik, I., Andronova, A., \& Johnson, T. C. (1993). Complex refractive index of atmospheric dust aerosols. Atmospheric Environment, 27 A(16), $2495-2502$.

Spada, M., Jorba, O., Pérez García-Pando, C., Janjic, Z., \& Baldasano, J. M. (2013). Modeling and evaluation of the global sea-salt aerosol distribution: sensitivity to size-resolved and sea-surface temperature dependent emission schemes. Atmospheric Chemistry and Physics, 13, 11735-11755.

Tegen, I., \& Lacis, A. A. (1996). Modeling of particle size distribution and its influence on the radiative properties of mineral dust aerosol. Journal of Geophysical Research, 101(D14), 19237-19244.

Xia, X. A., Chen, H. B., Wang, P. C., Zhang, W. X., Goloub, P., Chatenet, B., ... Holben, B. N. (2006). Variation of column-integrated aerosol properties in a Chinese urban region. Journal of Geophysical Research, 111, D05204.

Yu, H., Kaufman, Y. J., Chin, M., Feingold, G., Remer, L. A., Anderson, T. L., ... Zhou, M. (2006). A review of measurement-based assessments of the aerosol direct radiative effect and forcing. Atmospheric Chemistry and Physics, 6, 613-666.

Zhao, C., Liu, X., Qian, Y., Yoon, J., Hou, Z., Lin, G., ... Bao, J. (2013). A sensitivity study of radiative fluxes at the top of atmosphere to cloud-microphysics and aerosol parameters in the community atmosphere model CAM5. Atmospheric Chemistry and Physics, 13, 10969-10987. 